\title{
Chemical differentiation in regions of high-mass star formation
}

\section{CS, dust, and $\mathrm{N}_{2} \mathrm{H}^{+}$in southern sources}

\author{
L. Pirogov ${ }^{1}$, I. Zinchenko ${ }^{1,2,3}$, P. Caselli ${ }^{4,5}$, and L. E. B. Johansson ${ }^{6}$ \\ 1 Institute of Applied Physics of the Russian Academy of Sciences, Ulyanova 46, 603950 Nizhny Novgorod, Russia \\ e-mail: pirogov@appl.sci-nnov.ru \\ 2 Nizhny Novgorod University, Gagarin av. 23, 603950 Nizhny Novgorod, Russia \\ 3 Helsinki University Observatory, Tähtitorninmäki, PO Box 14, 00014 University of Helsinki, Finland \\ 4 INAF - Osservatorio Astrofisico di Arcetri, Largo E. Fermi 5, 50125 Firenze, Italy \\ 5 Harvard-Smithsonian Center for Astrophysics, 60 Garden St., Cambridge, MA 02138, USA \\ 6 Onsala Space Observatory, 43992, Onsala, Sweden
}

Received 26 December 2005 / Accepted 25 July 2006

ABSTRACT

\begin{abstract}
Aims. Our goals are to compare the $\mathrm{CS}, \mathrm{N}_{2} \mathrm{H}^{+}$, and dust distributions in a representative sample of high-mass star-forming dense cores and to determine the physical and chemical properties of these cores.

Methods. We compare the results of CS(5-4) and $1.2 \mathrm{~mm}$ continuum mapping of twelve dense cores from the southern hemisphere presented in this work, in combination with our previous $\mathrm{N}_{2} \mathrm{H}^{+}(1-0)$ and $\mathrm{CS}(2-1)$ data. We use numerical modeling of molecular excitation to estimate physical parameters of the cores.

Results. Most of the maps have several emission peaks (clumps). The mean sizes of 17 clumps with counterparts in the continuum and CS are 0.30(0.06) pc (continuum) and 0.51(0.07) pc (CS). For the clumps with IRAS sources, we derived dust temperatures of 24-35 K, masses of 90-6900 $M_{\odot}$, molecular hydrogen column densities of $(0.7-12.0) \times 10^{23} \mathrm{~cm}^{-2}$ and luminosities of $(0.6-46.0) \times$ $10^{4} L_{\odot}$. The LVG densities towards CS peaks within the $50^{\prime \prime}$ beam $(0.56 \mathrm{pc}$ at $2.3 \mathrm{kpc}$, the average distance of our sample source $)$ vary from source to source in the range $(3-40) \times 10^{5} \mathrm{~cm}^{-3}$. Masses calculated from LVG densities are higher than the CS virial masses and masses derived from continuum data, implying small-scale clumpiness of the cores. The molecular abundances towards IRAS sources in eight objects are $X(C S)=(0.3-2.7) \times 10^{-9}$ and $X\left(\mathrm{~N}_{2} \mathrm{H}^{+}\right)=(0.3-4.4) \times 10^{-10}$. The CS and continuum maps were compared with each other and with the $\mathrm{N}_{2} \mathrm{H}^{+}(1-0)$ maps. For most of the objects, the CS and continuum peaks are close to the IRAS point source positions. The CS(5-4) intensities correlate with continuum fluxes per beam in all cases, but only in five cases with the $\mathrm{N}_{2} \mathrm{H}^{+}(1-0)$ intensities. The study of the spatial variations of molecular integrated intensity ratios to continuum fluxes per beam reveals that $I\left(\mathrm{~N}_{2} \mathrm{H}^{+}\right) / F_{12}$ ratios drop towards the CS peaks for most of the sources, which can be due to an $\mathrm{N}_{2} \mathrm{H}^{+}$abundance decrease. For CS (5-4), the $I(\mathrm{CS}) / F_{1.2}$ ratios show no clear trends with distance from the CS peaks, while such ratios drop for CS(2-1) towards these peaks. Possible explanations of these results are considered. The analysis of normalized velocity differences between CS and $\mathrm{N}_{2} \mathrm{H}^{+}$lines has not revealed indications of systematic motions towards CS peaks.
\end{abstract}

Key words. stars: formation - ISM: clouds - ISM: molecules - radio lines: ISM

\section{Introduction}

The first step in most studies of dense cores of molecular clouds is their identification, either by a visual inspection or by rather sophisticated numerical analysis of molecular line maps, and yet, the maps of "traditional" tracers of dense gas $\left(\mathrm{CS}, \mathrm{HCN}, \mathrm{HCO}^{+}\right.$, $\mathrm{NH}_{3}, \mathrm{~N}_{2} \mathrm{H}^{+}$, etc.) appear to be different from each other in many cases. Most distinct differences are often observed between $\mathrm{CS}$ and $\mathrm{N}_{2} \mathrm{H}^{+}$maps. In low-mass starless cores (e.g. Tafalla et al. 2002), the CS emission vanishes towards the core center, while the $\mathrm{N}_{2} \mathrm{H}^{+}$emission is still high in the interior regions. Some more turbulent starless clumps show strong CS emission in comparison with $\mathrm{N}_{2} \mathrm{H}^{+}$(e.g. Williams \& Myers 1999; Olmi et al. 2005). The optically thick CS lines in the latter case are usually asymmetric and blueshifted, implying that they are probably tracing infall motions.

Both these cases can be explained by time dependent chemical models including depletion molecular species onto grains (Bergin \& Langer 1997; Bergin et al. 1997; Li et al. 2002;
Shematovich et al. 2003; Aikawa et al. 2003), suggesting different evolutionary stages of clumps within the parent molecular cloud. In the cores with embedded stellar objects, especially those of high mass, the morphologies of molecular maps become more complicated. This is due to the overlapping of the emission coming from the quiescent gas with that from outflows and hot cores, where dust grain mantles evaporate because of their proximity to YSOs. The $\mathrm{N}_{2} \mathrm{H}^{+}$intensities towards these objects may drop, while CS peaks towards YSO positions (e.g. Ungerechts et al. 1997; Bottinelli \& Williams 2004). Thus, chemical models of starless clumps cannot be used to explain the observed differences between $\mathrm{CS}$ and $\mathrm{N}_{2} \mathrm{H}^{+}$maps in high-mass star-forming regions.

In the past decade, a large sample of high-mass star-forming cores associated with water masers have been mapped in the CS(2-1) line (Zinchenko et al. 1994, 1995, 1998). Many of these cores have subsequently been mapped in different molecular lines, including $\mathrm{N}_{2} \mathrm{H}^{+}(1-0)$ (Pirogov et al. 2003, hereafter Paper I). Thus, the maps can be compared and important 
Table 1. Source list.

\begin{tabular}{lcrc}
\hline \hline Source & $\begin{array}{r}\text { RA (2000) } \\
\left({ }^{\mathrm{h}}\right)\left({ }^{\mathrm{m}}\right)\left({ }^{\mathrm{s}}\right)\end{array}$ & $\begin{array}{r}\text { Dec (2000) } \\
\left({ }^{\circ}{ }^{\prime \prime}\right)\end{array}$ & $\begin{array}{c}D \\
(\mathrm{kpc})\end{array}$ \\
\hline G 264.28+1.48 & 085627.8 & -430505 & $1.4^{a}$ \\
G 265.14+1.45 & 085924.7 & -434522 & $1.7^{a}$ \\
G 267.94-1.06 & 085903.6 & -473047 & $0.7^{a}$ \\
G 268.42-0.85 & 090154.3 & -474359 & $1.3^{a}$ \\
G 269.11-1.12 & 090332.8 & -482839 & $2.6^{a}$ \\
G 270.26+0.83 & 091643.3 & -475636 & $2.6^{a}$ \\
G 285.26-0.05 & 103130.0 & -580207 & $4.7^{a}$ \\
G 291.27-0.71 & 111149.9 & -611814 & $2.7^{b}$ \\
G 294.97-1.73 & 113912.6 & -632847 & $1.2^{a}$ \\
G 305.36+0.15 & 131233.9 & -623738 & $4.2^{a}$ \\
G 316.77-0.02 & 144458.9 & -594829 & $3.1^{c}$ \\
G 345.01+1.80 & 165645.3 & -401403 & $2.1^{c}$ \\
G 345.41-0.94 & 170933.7 & -413552 & $2.8^{c}$ \\
G 351.41+0.64 & 172053.4 & -354700 & $1.7^{d}$ \\
\hline
\end{tabular}

${ }^{a}$ Zinchenko et al. (1995); ${ }^{b}$ Brand \& Blitz (1993); ${ }^{c}$ Juvela (1996); ${ }^{d}$ Neckel (1978).

quantities can be determined for these regions, such as density distribution and chemical composition. Nonetheless, in contrast to the $\mathrm{N}_{2} \mathrm{H}^{+}(1-0)$ lines, which are optically thin in most cases (Paper I), the CS(2-1) lines are most likely optically thick, and possible differences between maps in these lines could be connected both with optical depth effects and chemical differences.

In this paper we present the results of $1.2 \mathrm{~mm}$ dust continuum and $\mathrm{CS}(5-4)$ observations in several southern sources. Optically thin dust emission is known to trace the gas component in highdensity regions closely, and it is insensitive to molecular abundance variations and radiative transfer effects. This can be used to separate optical depth and chemical effects in molecular line maps.

The goal of the paper is to analyze the dust continuum and $\mathrm{CS}(5-4)$ data in comparison with our previous $\operatorname{CS}(2-1)$ and $\mathrm{N}_{2} \mathrm{H}^{+}(1-0)$ results, in order to get reliable information on the density and chemical structure of the cores. The paper also contains estimates of physical parameters including sizes and masses, as well as density distributions derived from CS(5-4) and $\mathrm{CS}(2-1)$ data and molecular abundances for several sources.

\section{Observations}

The sources were originally selected from the sample of highmass star-forming regions associated with water masers located in the southern hemisphere (Zinchenko et al. 1995; Juvela 1996) and from the dense core database of Jijina et al. (1999), according to the following criteria: presence of embedded clusters of stars detected in infrared and distances to the objects not greater than $5 \mathrm{kpc}$. If no data on clusters were available, we selected sources with high IR-luminosities $\left(L>10^{4} L_{\odot}\right)$, which can be an indirect indication of cluster (Jijina et al. 1999). In total, 14 sources were selected for $\mathrm{CS}(5-4)$ observations, twelve of them observed in continuum at $1.2 \mathrm{~mm}$ wavelength. Most of the sources have associated IRAS point sources. The source list with coordinates and distances is given in Table 1.

\subsection{The CS(5-4) observations}

Observations of the $\mathrm{CS}(5-4)$ line at $244.9 \mathrm{GHz}$ towards 14 southern sources were carried out with the 15-m SEST antenna in February 2001. The $\mathrm{N}_{2} \mathrm{H}^{+}(1-0)$ line at $93.2 \mathrm{GHz}$ was observed simultaneously, and the results for this line are reported in Paper I. The telescope's half-power beam width at the $\operatorname{CS}(5-4)$ frequency is about $22^{\prime \prime}$, and the main beam efficiency is 0.5 . The system temperature was $\sim 400-1500 \mathrm{~K}$, depending on the source elevation and weather conditions. Spectral analysis was done using an acousto-optical spectrum analyzer (2000 channels) split into two halves to measure the $\mathrm{N}_{2} \mathrm{H}^{+}(1-0)$ and the $\mathrm{CS}(5-4)$ lines simultaneously. The frequency resolution was $42.6 \mathrm{kHz}$, which corresponds to a velocity resolution of $0.052 \mathrm{~km} \mathrm{~s}^{-1}$ at the CS(5-4) frequency.

Pointing was regularly checked by $\mathrm{SiO}$ maser observations and was typically better than $5^{\prime \prime}$. Mapping was done with $20^{\prime \prime}$ grid spacing. The data processing included baseline subtraction (low-order polynomials) and Gaussian fitting.

\subsection{Observations of dust continuum emission}

In June 2003, twelve sample sources were mapped in dust continuum emission at $250 \mathrm{GHz}$ with the SEST antenna. G267.94 and G305.36 were excluded from the list of continuum observations because they had not been completed in $\mathrm{CS}(5-4)$ and $\mathrm{N}_{2} \mathrm{H}^{+}(1-0)$.

We used the 37-element SIMBA bolometer array. The $H P B W$ of a single element is about $24^{\prime \prime}$, and the separation between elements on the sky is $44^{\prime \prime}$. Mapping was done by scanning in azimuth, sampling the signal every $8^{\prime \prime}$. The scans were spaced by $8^{\prime \prime}$ in elevation. This gave the pixel size $8^{\prime \prime}$. Typical map sizes are $900^{\prime \prime} \times 1200^{\prime \prime}$. The raw data were converted by the simbaread software and the processing was done using the MOPSI package written by R. Zylka according to the instructions in the SIMBA Observer's Handbook (2003) ${ }^{1}$. The final rms noise level derived from regions on the maps without sources is $30-60 \mathrm{mJy}^{\text {beam }}{ }^{-1}$ for 9 sources. For three sources (G345.01, G345.41, and G351.41), the rms noise level is $160-180 \mathrm{mJy}$ beam $^{-1}$. The flux measurements in the final coadded map are believed to be correct within $20 \%$. Note that the continuum fluxes also include contribution from molecular lines lying within the bolometer bandwidth (mainly $\mathrm{CO}(2-1)$ ). Using the Braine et al. (1995) analysis combined with $\mathrm{CO}(1-0)$ intensities (Zinchenko et al. 1995), we estimate that such a contribution is not significant for any source in our sample.

\section{Results}

The CS(5-4) integrated intensity maps, together with continuum maps, are shown in Fig. 1. For comparison, the $\mathrm{N}_{2} \mathrm{H}^{+}(1-0)$ maps (Paper I) are also plotted. In general, the continuum and the CS(5-4) maps have similar structures, and their morphologies range from close to spherical-symmetry (e.g. G270.26) to complex clumpy structures with several emission peaks (e.g. G316.77). The IRAS sources are indicated by stars and the uncertainty ellipses corresponding to a $95 \%$ confidence level in positional accuracy are also shown. For most of the objects (except G269.11, G316.77 and, probably, G265.14, and G345.41) these ellipses overlap with the CS and dust $90 \%$ intensity contours, indicating a coincidence of CS, continuum peaks, and IRAS point source positions.

Offset coordinates of CS(5-4) integrated intensity peaks, the values of the integrated intensities towards these positions, and parameters of Gaussian fits (main beam temperature, velocity,

\footnotetext{
1 http://puppis.1s.eso.org/staff/simba/manual/simba/ index.html
} 

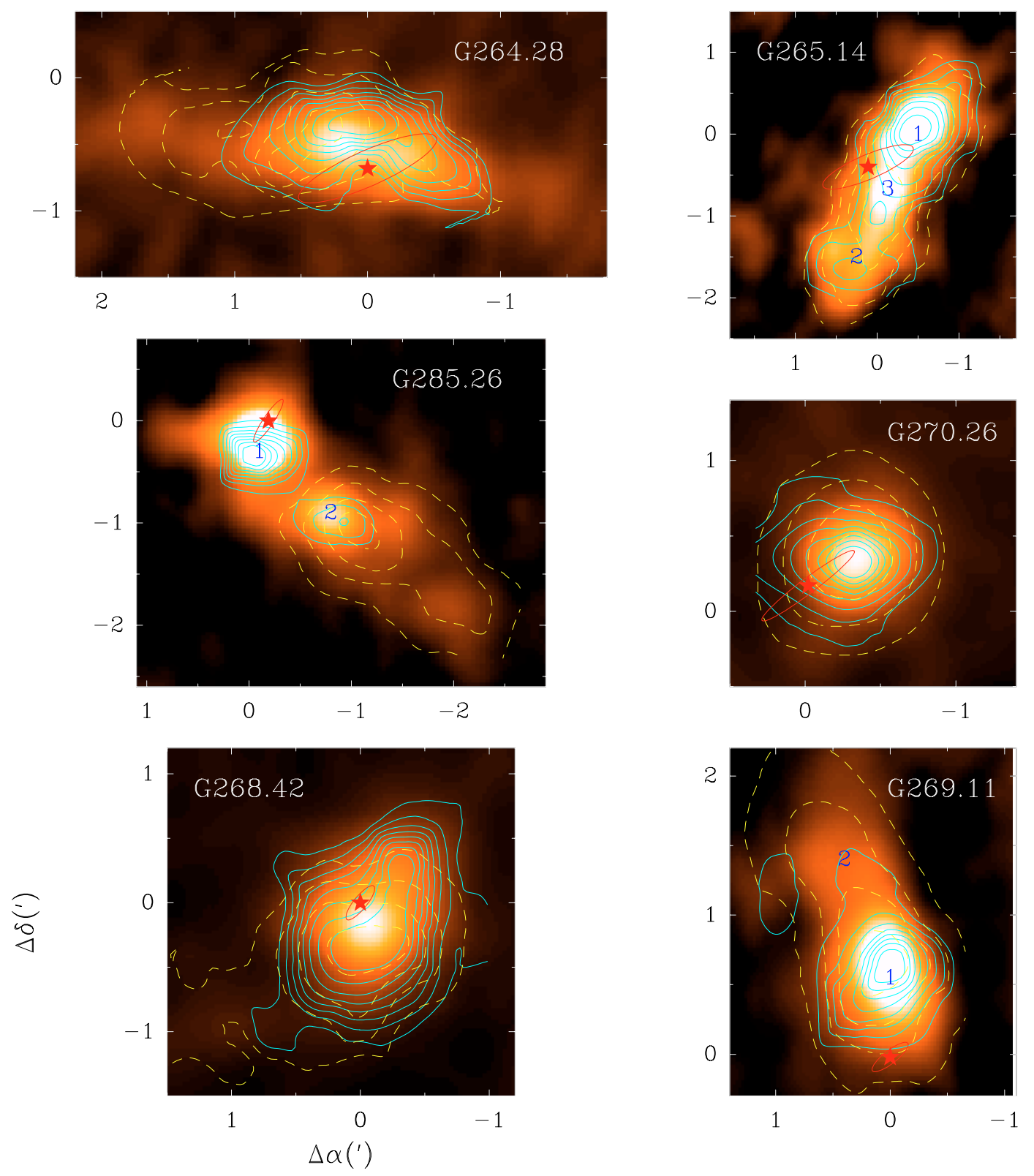

$0-1$

Fig. 1. Continuum $1.2 \mathrm{~mm}$ maps (in color) together with $\mathrm{CS}(5-4)$ (solid blue contours) and $\mathrm{N}_{2} \mathrm{H}^{+}(1-0)$ (dashed yellow contours, taken from Paper I) integrated intensity maps (color figures are available in the online version). Intensity contours range from $10 \%$ to $90 \%$ of the peak values with $10 \%$ step plus $5 \%$ contour (continuum), from $20 \%$ to $90 \%$ of the peak values with $10 \%$ step (CS), and from $30 \%$ to $90 \%$ of the peak values with $20 \%$ step $\left(\mathrm{N}_{2} \mathrm{H}^{+}\right)$. The CS peak integrated intensities are given in Table 2. The peak continuum fluxes (in Jy beam $\left.{ }^{-1}\right)$ are: $1.46(\mathrm{G} 264.28), 2.32$ (G265.14), 7.43 (G268.42), 3.44 (G269.11), 3.34 (G270.26), 4.39 (G285.26), 18.83 (G291.27), 1.64 (G294.97), 3.37 (G316.77), 3.93 (G345.01), 3.39 (G345.41), 12.54 (G351.41). The continuum clumps are marked by numbers as in Table 4. The IRAS point sources are marked by red stars. The uncertainty ellipses corresponding to $95 \%$ confidence level in the IRAS point-source position are also shown. The SEST beam size at the CS(5-4) frequency is shown on the G 264.28 map.

and line width) are given in Table 2 with the corresponding $1 \sigma$ errors, as defined by the fits, in parenthesis.

The CS(5-4) and continuum maps were deconvolved into individual clumps using our 2D Gaussian fitting program and the method described in Paper I. No attempts to separate individual clumps have been made in G264.28, where local emission peaks of nearly equal intensities are located too close to each other. We did not process the CS(5-4) map in G267.94 as it had not been completed. The parameters of individual CS(5-4) clumps, including relative coordinates of clump centers, aspect ratios (the ratios of the extents of the fitted elliptical Gaussians), and deconvolved angular $(\Delta \Theta)$ and linear $(d)$ sizes estimated as the geometric mean of the extents of the elliptical Gaussians at the half-maximum intensity level are given in Table 3 (Cols. 2-6). The clumps that belong to the same object are marked by numbers.

In Cols. 7 and 8 of Table 3 , the $\mathrm{CS}(5-4)$ mean line widths and virial masses are given. For homogeneous sphericallysymmetric clumps with no external pressure and no magnetic field, virial masses are given by:

$M_{\mathrm{vir}}\left(M_{\odot}\right)=105\langle\Delta V\rangle^{2} \cdot d$,

where $\langle\Delta V\rangle$ is the CS(5-4) mean line width (in $\mathrm{km} \mathrm{s}^{-1}$ ), defined as the weighted average of line widths at different positions within half-maximum intensity region; $d$ is the $\operatorname{CS}(5-4)$ emission region size in pc. Virial masses calculated according to the 

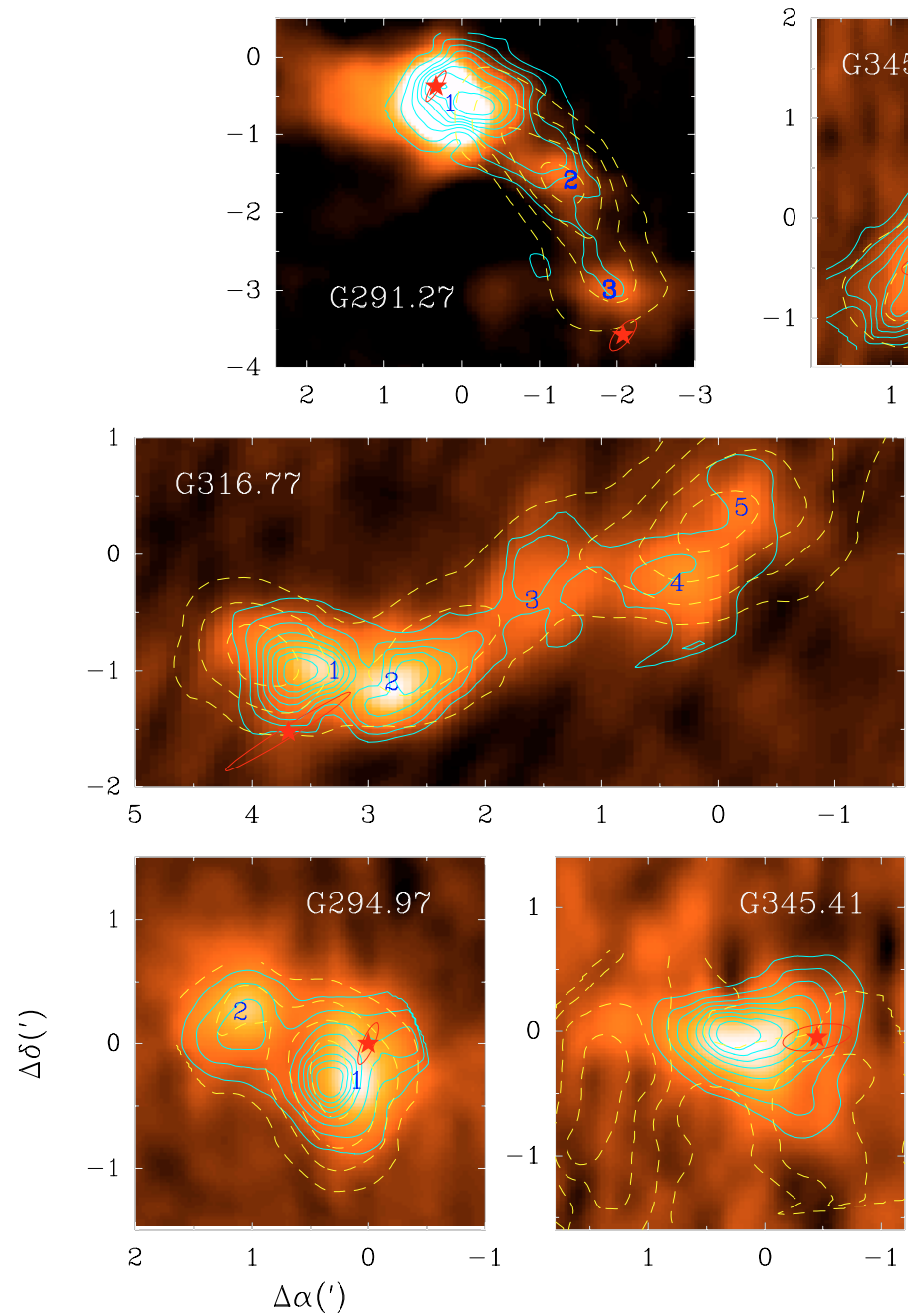
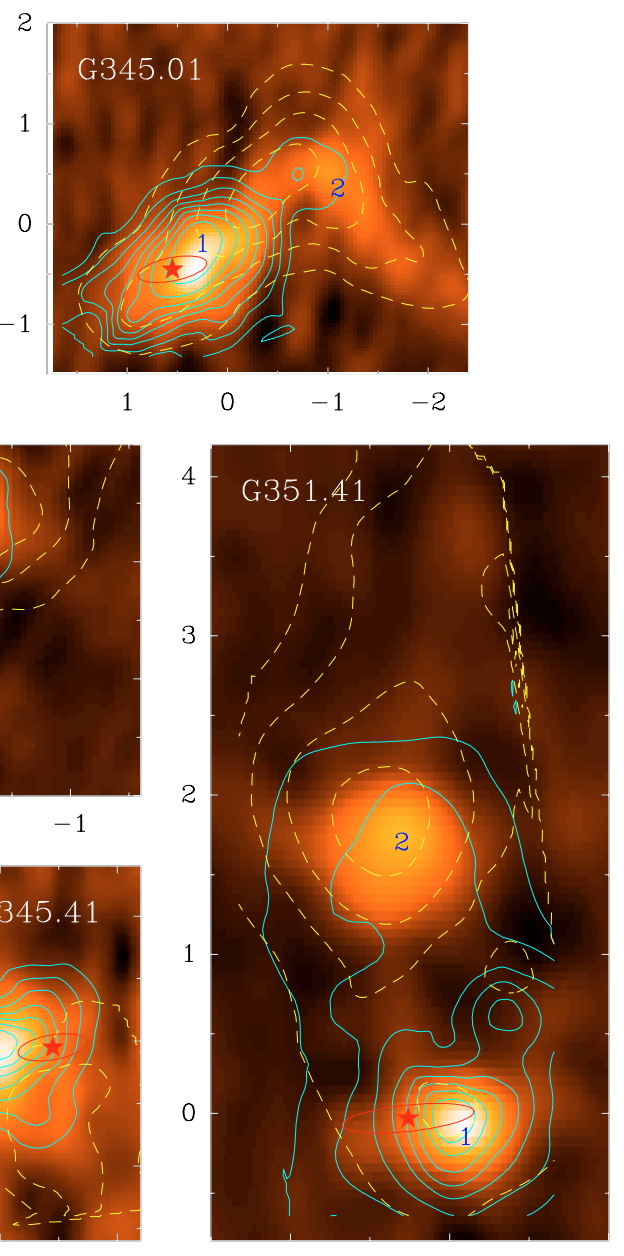

0

1

Fig. 1. continued.

Table 2. The CS(5-4) line parameters.

\begin{tabular}{lrrrrr}
\hline \hline Source & $\left(\Delta \alpha^{\prime \prime}, \Delta \delta^{\prime \prime}\right)$ & $\begin{array}{r}I \\
\left(\mathrm{~K} \mathrm{~km} \mathrm{~s}^{-1}\right)\end{array}$ & $\begin{array}{r}T_{\mathrm{MB}} \\
(\mathrm{K})\end{array}$ & $\begin{array}{r}V_{\mathrm{LSR}} \\
\left(\mathrm{km} \mathrm{s}^{-1}\right)\end{array}$ & $\begin{array}{r}\Delta V \\
\left(\mathrm{~km} \mathrm{~s}^{-1}\right)\end{array}$ \\
\hline G 264.28+1.48 & $(0,-20)$ & $13.3(0.2)$ & $5.08(0.06)$ & $5.93(0.01)$ & $2.34(0.03)$ \\
G 265.14+1.45 & $(-20,0)$ & $20.9(0.6)$ & $7.34(0.18)$ & $7.80(0.03)$ & $2.58(0.08)$ \\
G 267.94-1.06 & $(0,20)$ & $16.8(1.1)$ & $4.01(0.21)$ & $3.33(0.11)$ & $4.37(0.27)$ \\
G 268.42-0.85 & $(0,-20)$ & $30.8(0.7)$ & $10.26(0.22)$ & $3.51(0.03)$ & $2.90(0.07)$ \\
G 269.11-1.12 & $(0,40)$ & $35.8(0.7)$ & $5.82(0.11)$ & $9.74(0.05)$ & $5.78(0.13)$ \\
G 270.26+0.83 & $(-20,20)$ & $25.3(0.7)$ & $6.01(0.17)$ & $9.67(0.06)$ & $4.17(0.14)$ \\
G 285.26-0.05 & $(0,-20)$ & $31.8(0.3)$ & $5.76(0.05)$ & $3.67(0.02)$ & $5.04(0.05)$ \\
G 291.27-0.71 & $(-20,-40)$ & $43.0(0.6)$ & $10.44(0.14)$ & $-23.75(0.03)$ & $4.08(0.07)$ \\
G 294.97-1.73 & $(20,-20)$ & $14.9(0.5)$ & $4.71(0.17)$ & $-8.01(0.05)$ & $2.78(0.12)$ \\
G 305.36+0.15 & $(20,20)$ & $18.4(0.5)$ & $3.66(0.08)$ & $-38.56(0.05)$ & $5.46(0.14)$ \\
G 316.77-0.02 & $(220,-60)$ & $22.0(0.3)$ & $6.10(0.08)$ & $-38.44(0.02)$ & $3.41(0.05)$ \\
G 345.01+1.80 & $(20,-20)$ & $44.1(0.5)$ & $8.03(0.08)$ & $-13.48(0.03)$ & $5.32(0.06)$ \\
G 345.41-0.94 & $(20,0)$ & $69.2(0.5)$ & $13.87(0.10)$ & $-21.16(0.02)$ & $4.82(0.04)$ \\
G 351.41+0.64 & $(0,0)$ & $116.8(0.6)$ & $17.19(0.08)$ & $-7.03(0.01)$ & $6.42(0.04)$ \\
\hline
\end{tabular}

above formula lie in the range $\sim 140-1630 M_{\odot}$, with a mean value of $753 M_{\odot}$. We point out that these values can be overestimated, given that the CS(5-4) lines can be broadened due to optical depth. Shirley et al. (2003), who studied a large sample of high-mass star-forming regions in the northern hemisphere, found that $\mathrm{CS}(5-4)$ line widths are on average 1.3 times higher than those of presumably optically thin $C^{34} S(5-4)$ lines. In addition, if density in the cores decreases outwards, virial mass should be multiplied by a factor $\frac{3(5-2 p)}{5(3-p)}$, where $p$ is the power-law index for the density radial profile. For $p$ close to 2 (Paper I), this factor is close to 0.6.

The Cols. 2-6 of Table 4 contain parameters of continuum clumps similar to those of CS clumps. Total fluxes $\left(F_{\text {total }}\right)$ are given in Col. 7. The presence or an absence of an IRAS point source within the half maximum intensity level of individual clumps is marked by plus or minus signs in the last column of Tables 3 and 4. 
Table 3. Physical parameters of the CS(5-4) clumps.

\begin{tabular}{lrrrrrrrr}
\hline \hline Source & $\begin{array}{r}\Delta \alpha \\
\left({ }^{\prime \prime}\right)\end{array}$ & $\begin{array}{r}\Delta \delta \\
\left({ }^{\prime \prime}\right)\end{array}$ & $\begin{array}{r}\text { Aspect } \\
\text { ratio }\end{array}$ & $\begin{array}{r}\Delta \Theta \\
\left({ }^{\prime \prime}\right)\end{array}$ & $\begin{array}{r}d \\
(\mathrm{pc})\end{array}$ & $\begin{array}{r}\langle\Delta V\rangle \\
\left(\mathrm{km} \mathrm{s}^{-1}\right)\end{array}$ & $\begin{array}{r}M_{\text {vir }} \\
\left(M_{\odot}\right)\end{array}$ & IRAS \\
\hline G 264.28+1.48 & $4(3)$ & $-26(1)$ & $3.2(0.8)$ & $42(4)$ & $0.28(0.03)$ & $2.2(0.2)$ & 142 & + \\
G 265.14+1.45(1) & $-29(1)$ & $0(1)$ & $1.8(0.1)$ & $58(2)$ & $0.48(0.02)$ & $2.2(0.2)$ & 238 & + \\
G 265.14+1.45(2) & $12(2)$ & $-96(1)$ & $1.6(0.2)$ & $57(4)$ & $0.47(0.03)$ & $1.7(0.1)$ & 141 & - \\
G 268.42-0.85 & $-6(1)$ & $-12(2)$ & $1.6(0.1)$ & $64(3)$ & $0.40(0.02)$ & $2.7(0.2)$ & 307 & + \\
G 269.11-1.12 & $4(2)$ & $35(2)$ & $1.2(0.2)$ & $46(4)$ & $0.58(0.05)$ & $2.9(0.3)$ & 506 & - \\
G 270.26+0.83 & $-15(2)$ & $20(1)$ & $1.5(0.2)$ & $38(3)$ & $0.48(0.04)$ & $2.6(0.3)$ & 337 & + \\
G 285.26-0.05(1) & $-7(1)$ & $-20(1)$ & $1.2(0.2)$ & $18(2)$ & $0.42(0.04)$ & $4.5(0.3)$ & 868 & + \\
G 285.26-0.05(2) & $-49(2)$ & $-58(1)$ & $1.9(0.3)$ & $34(3)$ & $0.78(0.06)$ & $3.4(0.2)$ & 953 & - \\
G 291.27-0.71 & $-8(2)$ & $-31(1)$ & $1.4(0.1)$ & $70(2)$ & $0.92(0.03)$ & $4.0(0.1)$ & 1550 & + \\
G 294.97-1.73(1) & $16(3)$ & $-13(3)$ & $1.3(0.3)$ & $51(5)$ & $0.29(0.03)$ & $2.2(0.2)$ & 152 & + \\
G 294.97-1.73(2) & $54(7)$ & $0(3)$ & $1.6(0.5)$ & $63(9)$ & $0.37(0.06)$ & $2.4(0.2)$ & 219 & - \\
G 305.36+0.15 & $15(2)$ & $33(3)$ & $1.6(0.5)$ & $37(5)$ & $0.76(0.11)$ & $3.7(0.3)$ & 1090 & - \\
G 316.77-0.02(1) & $216(1)$ & $-59(1)$ & $1.1(0.2)$ & $32(3)$ & $0.48(0.04)$ & $3.9(0.3)$ & 762 & + \\
G 316.77-0.02(2) & $156(2)$ & $-64(2)$ & $2.7(0.6)$ & $32(4)$ & $0.49(0.06)$ & $4.4(0.2)$ & 973 & + \\
G 316.77-0.02(3) & $93(4)$ & $-17(5)$ & $2.1(0.6)$ & $62(9)$ & $0.93(0.14)$ & $4.1(0.4)$ & 1630 & - \\
G 316.77-0.02 (4) & $17(3)$ & $-12(2)$ & $1.5(0.3)$ & $57(6)$ & $0.85(0.09)$ & $3.3(0.3)$ & 985 & - \\
G 316.77-0.02(5) & $-14(3)$ & $38(5)$ & $2.2(1.0)$ & $39(8)$ & $0.59(0.13)$ & $3.7(0.3)$ & 870 & - \\
G 345.01+1.80 & $22(2)$ & $-23(1)$ & $1.9(0.1)$ & $85(2)$ & $0.87(0.02)$ & $4.0(0.1)$ & 1450 & + \\
G 345.41-0.94 & $4(2)$ & $-2(1)$ & $1.8(0.2)$ & $59(3)$ & $0.81(0.04)$ & $3.6(0.2)$ & 1070 & + \\
G 351.41+0.64 & $-3(1)$ & $-5(1)$ & $1.1(0.1)$ & $35(2)$ & $0.29(0.02)$ & $5.2(0.1)$ & 814 & + \\
\hline
\end{tabular}

Table 4. Physical parameters of dust clumps.

\begin{tabular}{|c|c|c|c|c|c|c|c|}
\hline Source & $\begin{array}{l}\Delta \alpha \\
\left({ }^{\prime \prime}\right)\end{array}$ & $\begin{array}{l}\Delta \delta \\
\left({ }^{\prime \prime}\right)\end{array}$ & $\begin{array}{l}\text { Aspect } \\
\text { ratio }\end{array}$ & $\begin{array}{l}\Delta \Theta \\
\left({ }^{\prime \prime}\right)\end{array}$ & $\begin{array}{r}d \\
(\mathrm{pc})\end{array}$ & $\begin{array}{r}F_{\text {total }} \\
(\mathrm{Jy})\end{array}$ & IRAS \\
\hline G 264.28+1.48 & $2.2(0.4)$ & $-33.0(0.2)$ & $4.0(0.2)$ & $36(1)$ & $0.24(0.01)$ & 5.0 & + \\
\hline G 265.14+1.45 (1) & $-28.1(0.3)$ & $3.2(0.3)$ & $1.4(0.1)$ & $30(1)$ & $0.25(0.01)$ & 5.1 & + \\
\hline G $265.14+1.45$ (2) & 14.4(0.6) & $-88.7(0.6)$ & $1.3(0.1)$ & $50(1)$ & $0.42(0.01)$ & 5.8 & - \\
\hline G $265.14+1.45$ (3) & $-8.1(0.3)$ & $-38.6(0.5)$ & $1.5(0.1)$ & $36(1)$ & $0.30(0.01)$ & 5.0 & + \\
\hline G 268.42-0.85 & $-5.9(0.2)$ & $-6.9(0.2)$ & $1.37(0.03)$ & $38.2(0.4)$ & $0.241(0.003)$ & 24.8 & + \\
\hline G 269.11-1.12 (1) & $1.1(0.2)$ & $34.5(0.2)$ & $1.6(0.1)$ & $27(1)$ & $0.34(0.01)$ & 7.5 & - \\
\hline G 269.11-1.12 (2) & $24(1)$ & $82(1)$ & $1.4(0.1)$ & $44(2)$ & $0.56(0.03)$ & 2.9 & - \\
\hline G $270.26+0.83$ & $-21.6(0.2)$ & $20.9(0.2)$ & $1.2(0.1)$ & $20.8(0.4)$ & $0.26(0.01)$ & 6.6 & + \\
\hline G 285.26-0.05 (1) & $-6.7(0.1)$ & $-11.3(0.1)$ & $1.03(0.03)$ & $19.8(0.3)$ & $0.45(0.01)$ & 7.0 & + \\
\hline G 285.26-0.05 (2) & $-48.3(0.4)$ & $-56.7(0.3)$ & $1.5(0.4)$ & $12(2)$ & $0.27(0.03)$ & 1.7 & - \\
\hline G 291.27-0.71 (1) & $11.0(0.1)$ & $-36.2(0.1)$ & $1.28(0.02)$ & $41.9(0.3)$ & $0.548(0.004)$ & 74.5 & + \\
\hline G 291.27-0.71 (2) & $-74(1)$ & $-92(1)$ & $2.5(0.2)$ & $45(2)$ & $0.59(0.03)$ & 6.2 & - \\
\hline G 291.27-0.71 (3) & $-121(1)$ & $-183(1)$ & $1.1(0.1)$ & $50(1)$ & $0.65(0.02)$ & 5.0 & - \\
\hline G 294.97-1.73 (1) & $6.4(0.4)$ & $-12.5(0.4)$ & $1.4(0.1)$ & $44(1)$ & $0.254(0.004)$ & 5.9 & + \\
\hline G 294.97-1.73 (2) & $65(1)$ & $15.7(0.4)$ & $1.1(0.1)$ & $42(1)$ & $0.24(0.01)$ & 3.9 & - \\
\hline G 316.77-0.02 (1) & 200(1) & $-61.8(0.3)$ & $2.7(0.1)$ & $46(1)$ & $0.69(0.01)$ & 9.1 & + \\
\hline G 316.77-0.02 (2) & $168(1)$ & $-66.2(0.4)$ & $2.5(0.2)$ & 41(1) & $0.62(0.02)$ & 8.7 & + \\
\hline G 316.77-0.02 (3) & $95(1)$ & $-24(1)$ & $1.8(0.1)$ & $56(1)$ & $0.84(0.02)$ & 6.2 & - \\
\hline G 316.77-0.02 (4) & $21(1)$ & $-15(1)$ & $1.1(0.1)$ & $40(1)$ & $0.60(0.02)$ & 5.7 & - \\
\hline G 316.77-0.02 (5) & $-14(1)$ & $25(1)$ & $1.3(0.1)$ & $39(2)$ & $0.59(0.02)$ & 3.6 & - \\
\hline G $345.01+1.80(1)$ & $15(1)$ & $-22.0(0.4)$ & $2.4(0.1)$ & $42(1)$ & $0.43(0.01)$ & 14.3 & + \\
\hline G 345.01+1.80 (2) & $-69(1)$ & $22(1)$ & $1.9(0.2)$ & $48(2)$ & $0.49(0.02)$ & 6.6 & - \\
\hline G 345.41-0.94 & $10(1)$ & $-7.7(0.4)$ & $2.3(0.1)$ & $42(1)$ & $0.57(0.02)$ & 12.6 & + \\
\hline G 351.41+0.64 (1) & $-5.2(0.3)$ & $-5.5(0.2)$ & $2.1(0.2)$ & $16(1)$ & $0.13(0.01)$ & 19.9 & + \\
\hline G 351.41+0.64 (2) & $20.3(0.3)$ & $99.8(0.3)$ & $1.05(0.04)$ & $32(1)$ & $0.26(0.01)$ & 21.8 & - \\
\hline
\end{tabular}

All the CS(5-4) clumps have corresponding counterparts in the continuum. Their centers coincide within $10^{\prime \prime}$ in most cases. Larger angular distances (up to $16^{\prime \prime}$ ) between CS and continuum clump centers have been found in G294.97(2) and G316.77(1); however, the sensitivity of the CS data is rather low in these cases. No attempts to reveal individual clumps were made in the case of G264.28 (both for continuum and CS maps) and G291.27 (for CS map) where the emission peaks are too close to each other and have comparable intensities.

In several cases (G265.14, G285.26, G291.27, G345.01, and G351.41), the continuum maps show more details than their $\mathrm{CS}(5-4)$ counterparts probably due to a higher signal-to-noise ratio, better spatial sampling, and the absence of optical depth effects. In G351.41, the continuum map reveals two clumps separated by about $110^{\prime \prime}$ in the north-south direction, which are associated with the well-known massive star-forming regions NGC 6334 I and NGC 6334 I(N), thought to be at different evolutionary stages (e.g. McCutcheon et al. 2000). Although the northern clump in our CS(5-4) map is not as prominent as in continuum, the map shows similar morphology and is in general agreement with the CS(7-6) results from McCutcheon et al. (2000).

Angular and linear sizes of $20 \mathrm{CS}$ clumps lie in the ranges $\Delta \Theta=18-85^{\prime \prime}$ and $d=0.28-0.93 \mathrm{pc}$ with $\langle\Delta \Theta\rangle=50^{\prime \prime}\left(6^{\prime \prime}\right)$ and 
$\langle d\rangle=0.52(0.07) \mathrm{pc}$; the numbers in parenthesis are rms deviations from the mean. The sizes of 25 nearby continuum clumps are: $\Delta \Theta=12-56^{\prime \prime}$ and $d=0.13-0.84 \mathrm{pc}$ with $\langle\Delta \Theta\rangle=33(4)^{\prime \prime}$ and $\langle d\rangle=0.34(0.06) \mathrm{pc}$. The continuum clumps in most of the sources are smaller than the CS clumps. For 17 clumps detected both in the continuum and CS (excluding G264.28 and G291.27) $\langle d\rangle=0.30(0.06) \mathrm{pc}$ (continuum) and 0.51(0.07) pc (CS). The difference in sizes could be connected with low signal-to-noise ratios and undersampling of the $\mathrm{CS}$ maps that may produce enlarged CS clumps sizes. Higher signal-to-noise ratios in the continuum observations allowed us to reveal multiple clumps in some sources (e.g. G269.11, G291.27), while in CS we see a single large clump. Still, it is also possible that the differences in sizes could be due to the CS opacity or abundance gradients within clumps. The CS clump sizes are close to those of $\mathrm{N}_{2} \mathrm{H}^{+}$ones (Paper I). Aspect ratios for CS and continuum clumps are: $1.1-3.2$ and $1.0-4.0$ with mean values $1.5(0.1)$ and 1.3(0.2), respectively. This parameter could be used for selecting nearly circular cores and exploring radial dependences of physical parameters within the clumps. Such an analysis lies beyond the scope of the present paper, so we postpone it to future publications.

\section{Physical parameters derived from continuum data and from LVG modeling}

Using our $1.2 \mathrm{~mm}$ data and IRAS fluxes, it is possible to estimate the physical parameters of the clumps including dust temperatures, masses, hydrogen column densities, and luminosities. The CS(5-4) data, together with the (2-1) data (Zinchenko et al. 1995), allowed us to calculate LVG densities, CS column densities, and molecular abundances for eight sources.

\subsection{Dust temperatures, masses, hydrogen column densities, and luminosities}

For the clumps with nearby IRAS sources, we have derived dust color temperatures by fitting the IRAS and $1.2 \mathrm{~mm}$ total fluxes $\left(F_{\text {total }}\right.$, Table 4$)$ with a 2-temperature greybody curve and by assuming optically thin conditions: $A_{1} v^{\beta} B_{v}\left(T_{1}\right)+A_{2} v^{\beta} B_{v}\left(T_{2}\right)$ (Mozurkewich et al. 1986). We exclude G269.11 from the analysis because the IRAS source lies outside the half-maximum level in this source. We set the power-law index of dust emissivityfrequency dependence $(\beta)$ equal to 2 in our calculations. Setting $\beta=1$ leads to a $10-20 \%$ increase in dust temperatures. The values of dust temperatures ( $T_{\mathrm{d}}$, cold component) are given in Col. 2, Table 5. The temperatures of the hot dust component lie in the range $\sim 95-115 \mathrm{~K}$. Dust masses were calculated according to the expression (e.g. Doty \& Leung 1994):

$M_{\mathrm{d}}=\frac{F_{\text {total }} D^{2}}{k_{1.2} B_{1.2}\left(T_{\mathrm{d}}\right)}$,

where $D, k_{1.2}$, and $B_{1.2}\left(T_{\mathrm{d}}\right)$ are the source distance, the dust mass absorption coefficient, and Plank function at $1.2 \mathrm{~mm}$, respectively. Ossenkopf \& Henning (1994) derived a mass absorption coefficient of $1 \mathrm{~cm}^{2} \mathrm{~g}^{-1}$ at $1.3 \mathrm{~mm}$ wavelength appropriate for cold dust grains covered with thick icy mantles. We adopted this value for our calculations assuming that water ice cannot evaporate at the derived values of $T_{\mathrm{d}}$ (e.g. van Dishoeck 2004). Gas masses $\left(M_{\mathrm{g}}\right)$, calculated assuming a gas-to-dust mass ratio, $R_{m}=100$, lie in the range $\sim 90-6900 M_{\odot}$. Note that these values could be underestimated if the dust temperature decreases outwards as expected in the case of internal heating. Using the
Table 5. Physical parameters derived from continuum data.

\begin{tabular}{lrrrr}
\hline \hline Source & $T_{\mathrm{d}}(\mathrm{K})$ & $M_{\mathrm{g}}\left(M_{\odot}\right)$ & $L\left(L_{\odot}\right)$ & $N_{\mathrm{H}_{2}}\left(\mathrm{~cm}^{-2}\right)$ \\
\hline G 264.28+1.48 & 32 & 93 & $6.6 \times 10^{3}$ & $7.0 \times 10^{22}$ \\
G 265.14+1.45 & 30 & 624 & $5.8 \times 10^{4}$ & $1.2 \times 10^{23}$ \\
G 268.42-0.85 & 35 & 360 & $4.6 \times 10^{4}$ & $3.2 \times 10^{23}$ \\
G 270.26+0.83 & 29 & 469 & $1.8 \times 10^{4}$ & $1.8 \times 10^{23}$ \\
G 285.26-0.05(1) & 33 & 1418 & $4.6 \times 10^{5}$ & $2.0 \times 10^{23}$ \\
G 291.27-0.71(1) & 25 & 6910 & $2.9 \times 10^{5}$ & $1.2 \times 10^{24}$ \\
G 294.97-1.73(1) & 27 & 98 & $5.9 \times 10^{3}$ & $9.6 \times 10^{22}$ \\
G 316.77-0.02 & 28 & 2415 & $1.9 \times 10^{5}$ & $1.8 \times 10^{23}$ \\
G 345.01+1.80(1) & 30 & 639 & $7.4 \times 10^{4}$ & $2.0 \times 10^{23}$ \\
G 345.41-0.94 & 29 & 1035 & $3.4 \times 10^{5}$ & $1.8 \times 10^{23}$ \\
G 351.41+0.64 (1) & 30 & 591 & $8.5 \times 10^{4}$ & $6.5 \times 10^{23}$ \\
\hline
\end{tabular}

simple model of an optically thin, spherical dust shell with radial temperature and density gradients (Pirogov \& Zinchenko 1998; see the Appendix) and taking the fluxes at $1.2 \mathrm{~mm}$ and $100 \mu \mathrm{m}$ for each source, it is possible to calculate the ratio of masses obtained in this model and in the isothermal approximation. It mainly depends on the ratio of outer-to-inner radii of the shell and the density-radius power-law index, increasing when these two parameters increase. After taking the power-law index $p=2$ and a radii ratio of $10^{4}$, we find that the mass of the shell for some sources can be up to 5 times higher than the mass calculated in the isothermal approximation.

Hydrogen column densities have been calculated from peak fluxes per beam and dust temperatures (e.g. Motte et al. 1998):

$N_{\mathrm{H}_{2}}=\frac{F_{\text {peak }}}{\Omega m R_{m}^{-1} k_{1.2} B_{1.2}\left(T_{\mathrm{d}}\right)}$,

where $\Omega$ is the beam solid angle, $m=2.33 \mathrm{amu}$ is mean molecular mass. The $N_{\mathrm{H}_{2}}$ values given in Col. 5 of Table 5 fall in the range $(0.7-12.0) \times 10^{23} \mathrm{~cm}^{-2}$.

The comparison of $M_{\mathrm{g}}$ masses with CS (Table 3) and $\mathrm{N}_{2} \mathrm{H}^{+}$virial masses (Paper I) for closely located clumps shows that they agree within a factor of 2 except for G291.27, where $M_{\mathrm{g}}$ exceeds the CS virial mass by a factor of 4.5. As the uncertainties in dust masses (mainly due to uncertainties in distance and in dust absorption coefficient and due to deviations from isothermal approximation) can be rather high, we conclude that the two mass estimates are in reasonable agreement.

Bolometric luminosities $(L)$ calculated by integrating the fitting curves over the total frequency range are given in Col. 4 of Table 5. They lie in the range $(0.6-46.0) \times 10^{4} L_{\odot}$, indicating the presence of embedded high-mass objects. The $L / M_{\mathrm{g}}$ ratio that is considered to be proportional to the star formation rate ranges from 38 to $325 L_{\odot} / M_{\odot}$ for the sources in Table 5 with a mean value $139 \pm 105 L_{\odot} / M_{\odot}$. This is consistent with $\langle L / M\rangle$ estimates for large samples of high-mass star-forming regions with masses obtained from dust continuum data: $71 \pm 56 L_{\odot} / M_{\odot}$ (Faundez et al. 2004), $136 \pm 100 L_{\odot} / M_{\odot}$ (Shirley et al. 2003), and $120 \pm 90 L_{\odot} / M_{\odot}$ (the data from Beuther et al. 2002 rescaled by Mueller et al. 2002).

\subsection{LVG densities and molecular abundances}

By taking the peak line intensities of two different CS transitions into account, one can derive hydrogen densities for any given kinetic temperature using the LVG approach. Nine sources of our sample were previously mapped in the $\mathrm{CS}(2-1)$ line with the SEST antenna (Zinchenko et al. 1995) with a 50" beam 
Table 6. Molecular column densities and abundances smoothed over $50^{\prime \prime}$ Gaussian beam towards positions close to IRAS sources.

\begin{tabular}{lrrrrrr}
\hline \hline $\begin{array}{l}\text { Source, } \\
\text { position }\end{array}$ & $\begin{array}{r}T_{\mathrm{KIN}} \\
(\mathrm{K})\end{array}$ & $\begin{array}{r}N\left(\mathrm{H}_{2}\right) \\
\left(\mathrm{cm}^{-2}\right) \\
\left(10^{22}\right)\end{array}$ & $\begin{array}{r}N(\mathrm{CS}) \\
\left(\mathrm{cm}^{-2}\right)\end{array}$ & $\begin{array}{r}X(\mathrm{CS}) \\
\left(10^{13}\right)\end{array}$ & $\begin{array}{r}N\left(\mathrm{~N}_{2} \mathrm{H}^{+}\right) \\
\left(\mathrm{cm}^{-2}\right)\end{array}$ & $\begin{array}{r}X\left(\mathrm{~N}_{2} \mathrm{H}^{+}\right) \\
\left(10^{-10}\right)\end{array}$ \\
\hline G 264.28 (0,-40) & 20 & 3.1 & 3.4 & 1.1 & 4.1 & 1.3 \\
G 265.14 (0,-20) & 20 & 4.7 & 4.8 & 1.0 & 16.1 & 3.4 \\
G 268.42 (0,0) & 40 & 13.8 & 14.0 & 1.0 & 8.7 & 0.6 \\
G 269.11 (0,0) & 20 & 3.4 & 4.5 & 1.3 & 14.0 & 4.2 \\
G 270.26 (0,0) & 40 & 2.9 & 7.9 & 2.7 & 13.0 & 4.4 \\
G 285.26 (0,0) & 40 & 6.0 & 4.4 & 0.7 & 1.6 & 0.3 \\
G 291.27 (20,-20) & 40 & 54.5 & 17.5 & 0.3 & 15.6 & 0.3 \\
G 294.97 (0,0) & 40 & 4.4 & 5.2 & 1.2 & 11.0 & 2.5 \\
\hline
\end{tabular}

and $40^{\prime \prime}$ grid spacing. The peak $\mathrm{CS}(2-1)$ positions were also observed in the $\mathrm{CO}(1-0)$ line by these authors. We compared their data with our CS(5-4) data for eight sources (except G267.94).

In order to use both the $\operatorname{CS}(2-1)$ and the $\operatorname{CS}(5-4)$ data in model calculations, it is important to convolve them to the same beam. For this purpose we smoothed the $\operatorname{CS}(5-4)$ main-beam temperature maps to the $50^{\prime \prime}$ resolution using our 2D Gaussian fitting program and calculated errors of the convolved $\mathrm{CS}(5-4)$ temperatures using the propagation-of-error approach. We applied the $\mathrm{CS}-\mathrm{H}_{2}$ collisional rates from Turner et al. (1992), which are tabulated for given kinetic temperatures. The model is isothermal, and kinetic temperatures were estimated according to the temperatures of the $\mathrm{CO}(1-0)$ line and of the dust (cold component) (Table 5). The assumed values of kinetic temperatures are given in Table 6. Twelve CS rotational levels were considered in the calculations.

The results of model calculations for eight analyzed sources are shown in Fig. 2 as density versus the projected distance from the positions of peak $\mathrm{CS}(5-4)$ integrated intensities (Table 2). Central densities vary in the range $(3-40) \times 10^{5} \mathrm{~cm}^{-3}$. In all but two sources (G270.26 and G294.97), density clearly decreases with distance from the CS(5-4) peaks. In four sources (G265.14, G268.42, G269.11, and G291.27), density falls by about an order of magnitude at $\sim 0.8-2 \mathrm{pc}$ from the center. The slopes of density-radius dependences for the outer regions of these sources vary from -2.0 to -3.6 . In G265.14 and G291.27, these trends may be due to the existence of neighboring low-density clumps (see Fig. 1). Note that the calculated LVG densities depend on the adopted kinetic temperatures (the lower the temperature, the higher the density). If the kinetic temperature decreases outwards, calculated densities far from the center could be underestimated. Changing the temperature from $40 \mathrm{~K}$ to $20 \mathrm{~K}$ leads to a density increase by about half an order of magnitude. Also, densities could be slightly overestimated towards the positions of IRAS sources due to the influence of infrared pumping on CS excitation (see Sect. 6).

If taking density estimates into account, it is possible to calculate gas masses for the most intensive CS(5-4) clumps in each source. Assuming spherically-symmetric clumps with radius $d / 2$, where $d$ is the CS(5-4) clump size (Table 3 ), the masses could be calculated as $M=4 \pi / 3 m\langle n\rangle(d / 2)^{3}$, where $\langle n\rangle$ is the density averaged over positions within the area of radius $d / 2$. Using these masses, we calculate mass ratios, $M_{\mathrm{vir}} / M$, that should be considered as dense gas volume-filling factor. The mean mass ratio for eight sources is $0.2 \pm 0.2$. This value is consistent with the one found by Shirley et al. (2003) for their sample of about 40 dense high-mass star-forming regions $(0.5 \pm 0.7)$. It is also consistent with the estimate $\lesssim 0.2$ for a dense gas volume-filling factor found by Juvela (1998) from
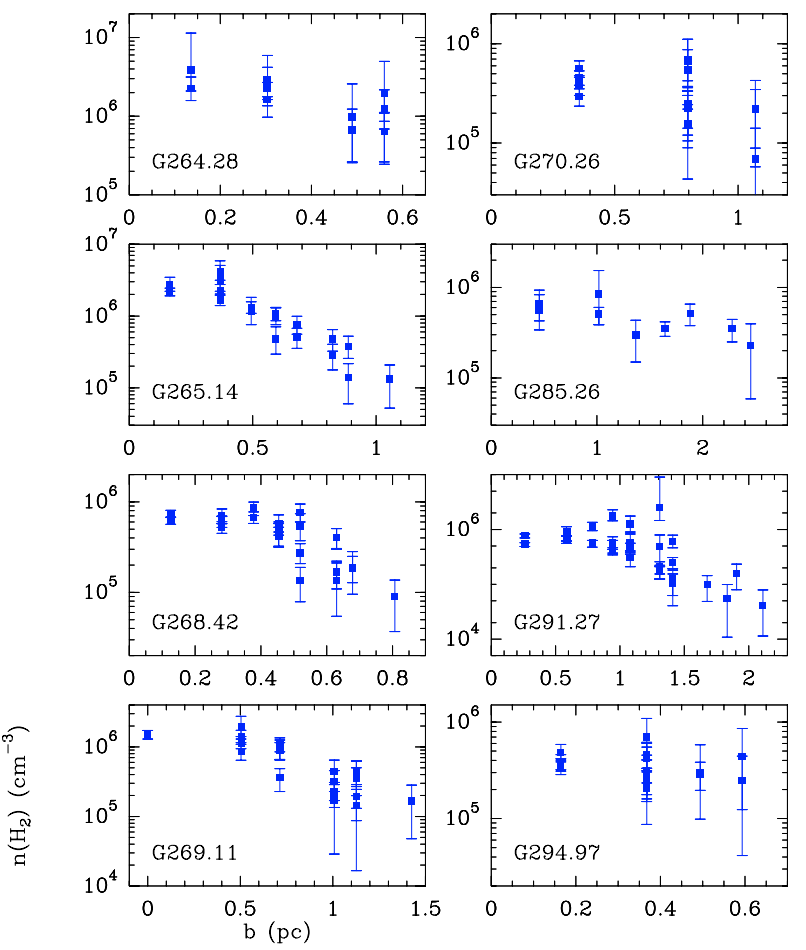

Fig. 2. LVG densities for eight sources versus projected distance from the $\mathrm{CS}(5-4)$ peaks. Error bars correspond to $1 \sigma$ uncertainty in the CS line temperatures.

multi-line $\mathrm{CS}$ and $\mathrm{C}^{34} \mathrm{~S}$ profile modeling towards a sample of southern high-mass star-forming regions.

Using CS column densities from LVG calculations and $\mathrm{H}_{2}$ column densities from $1.2 \mathrm{~mm}$ continuum data smoothed to the 50" beam, we calculated the CS abundances for eight sources towards positions close to IRAS sources. The calculated values are given in Table 6 . For the same positions, we calculated the $\mathrm{N}_{2} \mathrm{H}^{+}$LTE column densities (at $T_{\mathrm{EX}}=10 \mathrm{~K}$ ) and the $\mathrm{N}_{2} \mathrm{H}^{+}$abundances. The abundances lie in the following ranges $X(\mathrm{CS})=(0.3-2.7) \times 10^{-9}, X\left(\mathrm{~N}_{2} \mathrm{H}^{+}\right)=(0.3-4.4) \times 10^{-10}$. The lowest $X\left(\mathrm{~N}_{2} \mathrm{H}^{+}\right)$values were found towards the most luminous IRAS sources. Mean $\mathrm{N}_{2} \mathrm{H}^{+}$abundances for several clumps were calculated in Paper I using $\mathrm{N}_{2} \mathrm{H}^{+}$virial mass estimates. There is reasonable agreement between different $X\left(\mathrm{~N}_{2} \mathrm{H}^{+}\right)$estimates for clumps in G265.14, G269.11, and G294.97. For G268.42 our estimate of $X\left(\mathrm{~N}_{2} \mathrm{H}^{+}\right)$is about 4 times lower than the estimate from Paper I. For G285.26 and G291.27, our $X\left(\mathrm{~N}_{2} \mathrm{H}^{+}\right)$values are about an order of magnitude lower than the estimates from Paper I, but they relate to different clumps, implying abundance variations over the sources. The role of abundance variations is discussed in Sect. 6.

Note, that other physical parameters found for the sample cores (sizes, aspect ratios, densities, masses, dust temperatures, and luminosities) lie within the ranges found in the large surveys of high-mass star-forming regions performed during recent years in CS(5-4) (Plume et al. 1997; Shirley et al. 2003) and $1.2 \mathrm{~mm}$ continuum (Beuther et al. 2002; Mueller et al. 2002; Faundez et al. 2004; Fontani et al. 2005).

\section{Comparison of molecular and continuum data}

The maps in Fig. 1 show in many cases significant differences between $\mathrm{CS}(5-4)$ and $\mathrm{N}_{2} \mathrm{H}^{+}(1-0)$ intensity distributions, as well as between $1.2 \mathrm{~mm}$ continuum and $\mathrm{N}_{2} \mathrm{H}^{+}(1-0)$. We performed a 

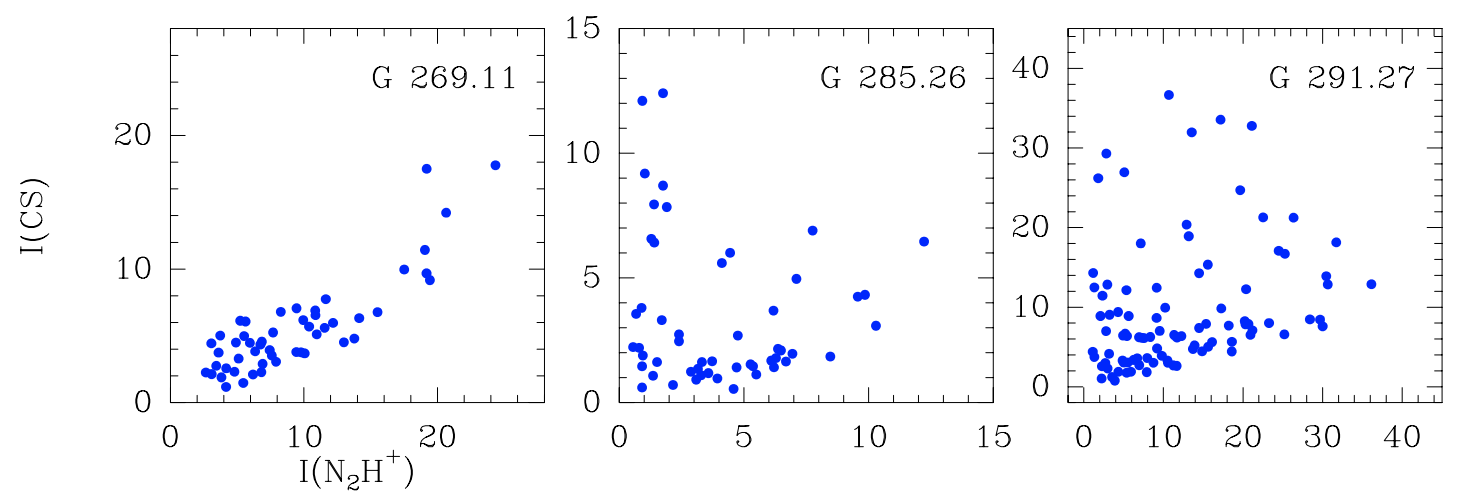

Fig. 3. The CS(5-4) versus $\mathrm{N}_{2} \mathrm{H}^{+}(1-0)$ integrated intensities (both in $\mathrm{K} \mathrm{km} \mathrm{s}^{-1}$ ) for three representative sources. The CS data is smoothed to the $50^{\prime \prime}$ resolution.

detailed point-to-point comparison of molecular intensities and continuum fluxes for each source and the results are given below. In addition, we compared the $\mathrm{CS}(5-4)$ and the $\mathrm{N}_{2} \mathrm{H}^{+}(1-0)$ line velocities and line widths.

\subsection{CS and dust maps}

The comparison of the CS(5-4) and $1.2 \mathrm{~mm}$ continuum data shows that in most cases the CS intensities depend almost linearly on continuum fluxes, implying that $\mathrm{CS}(5-4)$ is a good tracer of total gas column density. Some deviations from linear dependences could be connected with differences in excitation conditions in clumps within the same source.

\section{2. $\mathrm{CS}$ and $\mathrm{N}_{2} \mathrm{H}^{+}$maps}

In many cases (especially in G285.26, G291.27, G345.01, G345.41, and G351.41; see Fig. 1), the $\mathrm{N}_{2} \mathrm{H}^{+}(1-0)$ and the CS(5-4) intensities have different spatial distributions which, in general, could be caused by opacity effects, line excitation, or differences in the chemical composition of individual clumps.

For comparison with the $\mathrm{N}_{2} \mathrm{H}^{+}(1-0)$ data obtained with the $55^{\prime \prime}$ beam, the $\mathrm{CS}(5-4)$ integrated intensities were averaged over areas with $25^{\prime \prime}$ radius around each position. In five objects (G265.14, G268.42, G269.11, G270.26, and G294.97), $I(\mathrm{CS})$ depends almost linearly on $I\left(\mathrm{~N}_{2} \mathrm{H}^{+}\right)$. Other sources show a low correlation or no correlation. In some of them (G285.26, G345.01, and G351.41), the plots split into separate branches that probably belong to different clumps. In Fig. 3 the CS(5-4) integrated intensities are shown versus $I\left(\mathrm{~N}_{2} \mathrm{H}^{+}\right)$for three representative sources with high correlation (G269.11) and low correlation $(\mathrm{G} 285.26$ and $\mathrm{G} 291.27)$ between intensities.

Comparison of the $\mathrm{N}_{2} \mathrm{H}^{+}(1-0)$ maps with the $\mathrm{CS}(2-1)$ maps (Zinchenko et al. 1995) shows that they are in better agreement in some cases (G264.28, G285.26, and G291.27) than with the CS(5-4) maps.

\subsection{Intensity ratios}

To study spatial variations of molecular and continuum emission within the sources, we calculated the $\mathrm{CS}(5-4)$ to the $\mathrm{N}_{2} \mathrm{H}^{+}(1-0)$ integrated intensity ratios, as well as the ratios of molecular integrated intensities to continuum fluxes $\left(I(\mathrm{CS}) / F_{1.2}\right.$ and $\left.I\left(\mathrm{~N}_{2} \mathrm{H}^{+}\right) / F_{1.2}\right)$ for each source position. The continuum fluxes $\left(F_{1.2}\right)$ were averaged over areas with $25^{\prime \prime}$ radius around each $\mathrm{N}_{2} \mathrm{H}^{+}$position for $I\left(\mathrm{~N}_{2} \mathrm{H}^{+}\right) / F_{1.2}$ dependences. The ratios of molecular integrated intensities to continuum fluxes give information on abundance variations over the sources, if lines are optically thin, as in the case of $\mathrm{N}_{2} \mathrm{H}^{+}(1-0)$ (Paper I). We used data with signals higher than $3 \sigma$ and rejected map positions with intensities below 5\% the peak values (3\% for G268.42 and G291.27), to avoid the spikes of ratios that sometimes occur at the edges of the maps.

The dependences of $I(\mathrm{CS}) / I\left(\mathrm{~N}_{2} \mathrm{H}^{+}\right)$(upper panels), $I(\mathrm{CS}) / F_{1.2}$ (middle panels), and $I\left(\mathrm{~N}_{2} \mathrm{H}^{+}\right) / F_{1.2}$ (lower panels) with respect to projected distance from the $\mathrm{CS}(5-4)$ peaks $(b)$ are shown in Fig. 4 for three selected sources, where differences between the $\mathrm{CS}(5-4)$ and $\mathrm{N}_{2} \mathrm{H}^{+}(1-0)$ intensity distributions are among the most prominent. In several sources (especially in G285.26, G345.41, and G351.41), the $I(C S) / I\left(\mathrm{~N}_{2} \mathrm{H}^{+}\right)$ratios have a tendency to decrease with distance. Six sources demonstrate variations in $I(\mathrm{CS}) / I\left(\mathrm{~N}_{2} \mathrm{H}^{+}\right)$across the source by more than an order of magnitude (G285.26, G291.27, G345.01, $\mathrm{G} 345.41$, and G351.41). The highest ratios $(\sim 13-15)$ are observed in G285.26, G291.27, and G345.41, in the vicinity of the most luminous IRAS sources of our sample. The lowest ratios $(\$ 0.1)$ are observed in G316.77, G345.01, and G351.41 towards positions close to the centers of the $\mathrm{N}_{2} \mathrm{H}^{+}$clumps without CS counterparts and IRAS sources.

There is a rather large scatter both in $I(\mathrm{CS}) / F_{1.2}$ and $I\left(\mathrm{~N}_{2} \mathrm{H}^{+}\right) / F_{1.2}$ ratios across the sources (about an order of magnitude). The $I\left(\mathrm{~N}_{2} \mathrm{H}^{+}\right) / F_{1.2}$ ratios clearly drop towards CS peaks in most cases, while the $I(\mathrm{CS}) / F_{1.2}$ ratios demonstrate no such trends. No clear trends of $I\left(\mathrm{~N}_{2} \mathrm{H}^{+}\right) / F_{1.2}$ versus $b$ have been found in G268.42 and G294.97.

For several sources we calculated ratios of the CS(2-1) both to the $\mathrm{N}_{2} \mathrm{H}^{+}(1-0)$ integrated intensities and to continuum fluxes averaged over areas with $25^{\prime \prime}$ radii around each $\mathrm{CS}(2-1)$ position. It is found that these ratios show similar trends with $b$ as the $I(\mathrm{CS}) / I\left(\mathrm{~N}_{2} \mathrm{H}^{+}\right)$ratios for $\mathrm{CS}(5-4)$ and the $I\left(\mathrm{~N}_{2} \mathrm{H}^{+}\right) / F_{1.2}$ ratios (see plots for G285.26 in Fig. 4). For CS(2-1) an increase in the $I(\mathrm{CS}) / F_{1.2}$ ratios with $b$ is probably connected with line saturation (see Sect. 6 and Appendix A).

The comparison of intensity ratios at positions close to IRAS sources with bolometric luminosities (Table 5) shows that the $I(\mathrm{CS}) / I\left(\mathrm{~N}_{2} \mathrm{H}^{+}\right)$ratios are clearly enhanced towards the three most luminous sources (G285.26, G291.27, and G345.41) and the $I\left(\mathrm{~N}_{2} \mathrm{H}^{+}\right) / F_{1.2}$ ratios drop towards these sources.

\section{4. $\mathrm{CS}$ and $\mathrm{N}_{2} \mathrm{H}^{+}$velocities and line widths}

The velocity comparison of two molecular lines with different optical depths gives the information about the internal kinematics of the sources. Mardones et al. (1997) have introduced 

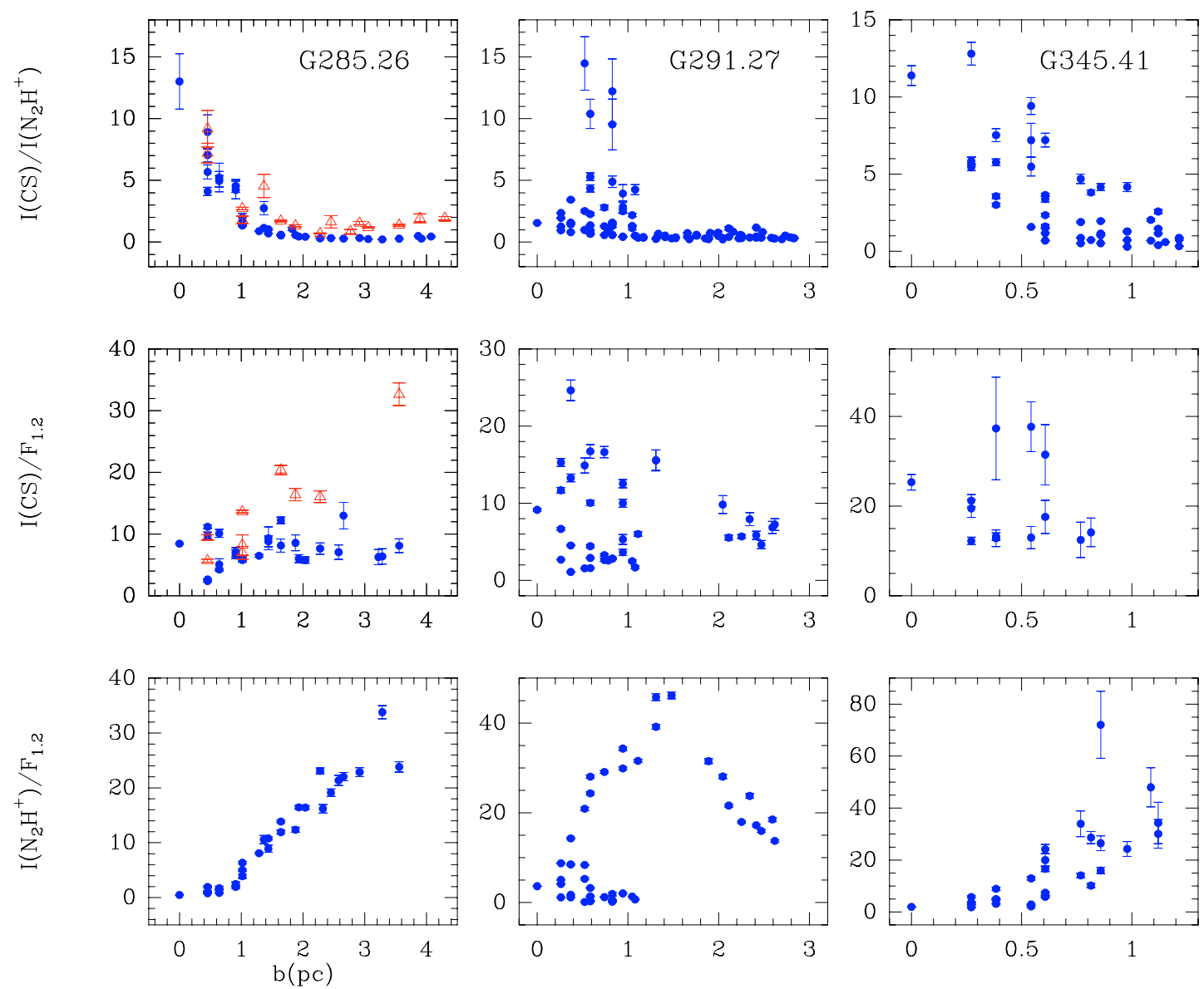

Fig. 4. The CS(5-4)/ $\mathrm{N}_{2} \mathrm{H}^{+}(1-0)$ integrated intensity ratios and the ratios of molecular intensities to continuum fluxes versus projected distance from the CS(5-4) peaks for three sources with the most prominent differences between $\mathrm{CS}$ and $\mathrm{N}_{2} \mathrm{H}^{+}$(in the lower panels, $F_{1.2}$ is the flux at $1.2 \mathrm{~mm}$ in Jy beam ${ }^{-1}$ averaged over areas with $25^{\prime \prime}$ radius around each $\mathrm{N}_{2} \mathrm{H}^{+}$position). In addition, the $\mathrm{CS}(2-1) / \mathrm{N}_{2} \mathrm{H}^{+}(1-0)$ and the CS(2-1)/continuum intensity ratios for G285.26 are shown by red triangles. In this case continuum fluxes have been averaged over areas with $25^{\prime \prime}$ radii around each $\mathrm{CS}(2-1)$ position. Error bars correspond to $1 \sigma$ uncertainties on the ratios.

normalized velocity difference: $\delta V=\left(V_{\text {thick }}-V_{\text {thin }}\right) / \Delta V_{\text {thin }}$, where $V_{\text {thick }}$ and $V_{\text {thin }}$ are optically thick and thin line velocities, respectively, and $\Delta V_{\text {thin }}$ an optically thin line width. Their analysis of molecular lines in low-mass cores has proved this parameter to be an effective tracer of infall (Mardones et al. 1997; Lee et al. 1999, 2001) and/or core accretion motions (e.g. Tafalla et al. 2002). Although $\mathrm{N}_{2} \mathrm{H}^{+}$and CS are likely to trace different regions, as in low-mass cores, it is still interesting to quantify the relative motions between different parts of the same cloud and see if there are signs of systematic contraction or expansion.

The $\mathrm{N}_{2} \mathrm{H}^{+}$lines most probably are optically thin in the sources (Paper I), while the CS lines could be optically thick. To search for systematic motions, we calculated normalized velocity differences between $\mathrm{CS}$ and $\mathrm{N}_{2} \mathrm{H}^{+}\left(\delta V_{\mathrm{CS}}\right)$ taken from the Gaussian fits for each source position. Both the CS(5-4) and CS(2-1) data (Zinchenko et al. 1995) were used. Given that the signal-to-noise ratios of the CS(5-4) data are rather low, we only analyzed the spectra with integrated intensities not lower than $20 \%-30 \%$ of the maximum value. Even if our spatial resolution does not permit resolving the regions with systematic motions, one could expect to detect non-zero normalized velocity differences towards positions close to density peaks.

According to the criterion $|\delta V|>0.25$ from Mardones et al. (1997), no indications of systematic motions have been found towards CS(5-4) peaks. However, we have found such indications towards some other positions in several sources. In particular, in $\mathrm{G} 285.26$ the $\delta V_{\mathrm{CS}}$ values towards the $\left(0^{\prime \prime}, 0^{\prime \prime}\right)$ position are $-0.58(0.15)$ and $-0.73(0.19)$ for CS(5-4) and CS $(2-1)$, respectively, probably implying infall motions.

Both $\mathrm{CS}$ and $\mathrm{N}_{2} \mathrm{H}^{+}$line widths are much higher than the thermal widths, and the line profiles in most sources are nearly symmetric without prominent self-absorption features. The CS (2-1) lines are broader than the $\mathrm{N}_{2} \mathrm{H}^{+}(1-0)$ lines in most cases (mean line width ratios vary from source to source in the range $0.9-1.7)$. The $\operatorname{CS}(5-4)$ lines are narrower than $\operatorname{CS}(2-1)$, and their widths are on average close to the $\mathrm{N}_{2} \mathrm{H}^{+}(1-0)$ widths implying that the CS(5-4) optical depths are not high (mean line width ratios vary in the range $0.7-1.3)$. In some sources (G269.11, G285.26, G291.27, G345.01, and G345.41), the CS(5-4) line widths are broader towards CS peaks and decrease outwards. Similar trends found in Paper I for the $\mathrm{N}_{2} \mathrm{H}^{+}(1-0)$ line widths were explained as an influence of enhanced dynamical activity in the central regions of the cores in the vicinity of IRAS sources. In the case of CS(5-4), these trends could also be connected with enhanced optical depth towards CS peaks.

The clumps where $\mathrm{N}_{2} \mathrm{H}^{+}$drops towards CS peaks (Sect. 5.3) have enhanced CS(5-4) mean line widths ( $\gtrsim 4 \mathrm{~km} \mathrm{~s}^{-1}$, Table 3), implying either enhanced optical depth or some dynamical processes that broaden line profiles towards these peaks. There is also a weak correlation between $\Delta V(\mathrm{CS})$ and luminosities for positions close to IRAS sources. 


\section{Discussion}

Here we examine possible reasons for the observed variations in the $\mathrm{CS} / \mathrm{N}_{2} \mathrm{H}^{+}, \mathrm{CS} /$ dust, and $\mathrm{N}_{2} \mathrm{H}^{+} /$dust ratios:

Opacity effects. The effect of line saturation due to opacity leads to a decrease in the relative line intensities towards CS and dust emission peaks (where column density is presumably the highest one). We have not seen such effect for the CS(5-4) data, and this can be considered as an implicit argument against high optical depth in these lines. On the other hand, a decrease in $\mathrm{CS}(2-1)$ relative intensities towards density peaks in several sources could be connected with saturation. Using the $C^{34}(2-1)$ data for several sample sources (Zinchenko et al. 1995), we find that the optical depth of the $\operatorname{CS}(2-1)$ line is higher than unity, implying a decrease in the $\mathrm{CS}(2-1)$ intensity compared with the optically thin case. For G285.26 $\left(-40^{\prime \prime},-40^{\prime \prime}\right), \tau=2.5$ and the $\mathrm{CS}(2-1)$ intensity decreases 2.7 times. The $\mathrm{N}_{2} \mathrm{H}^{+}(1-0)$ lines most probably are not optically thick (Paper I) within the $55^{\prime \prime}$ beam, and it is hard to consider opacity effects as an explanation of the observed variations on the scales resolved by the beam.

Excitation. Molecular line excitation is determined by collisions and radiative transfer processes. Differences in critical densities of the $\mathrm{CS}(5-4)$ and $\mathrm{N}_{2} \mathrm{H}^{+}(1-0)$ lines by about an order of magnitude can produce differences in their excitation and intensity distributions. The analysis given in Appendix A shows that density, kinetic, and dust temperature gradients can cause a decrease in the $I\left(\mathrm{~N}_{2} \mathrm{H}^{+}\right) / F_{1.2}$ ratios by a factor not more than 3 in the density range $10^{5}-10^{6} \mathrm{~cm}^{-3}$ when $T_{\text {KIN }}$ and $T_{\mathrm{d}}$ vary from $20 \mathrm{~K}$ to $40 \mathrm{~K}$. However, they cannot be the reason for the observed drops in these ratios by an order of magnitude or higher, as, for example, in G285.26 where high-density contrasts are not observed (Fig. 2) and in other sample sources.

In principle, the observed variations could be connected with radiative transfer effects caused by pumping from nearby luminous infrared sources that can overpopulate the upper rotational levels of the molecule and underpopulate the lower levels. This effect could in our case make the CS(5-4) line intensities higher and the $\mathrm{N}_{2} \mathrm{H}^{+}(1-0)$ line intensities lower compared with the case of no pumping. However, Carroll \& Goldsmith (1981) showed that for CS the size of the region affected by IR pumping via vibrational transitions is $\lesssim 0.03$ pc. We did a similar analysis for $\mathrm{N}_{2} \mathrm{H}^{+}$using parameters of vibrational transitions from Botschwina (1984), Owrutsky et al. (1986), and Heninger et al. (2003). It is found that the size of the affected region considering the bending mode is $\$ 0.02 \mathrm{pc}$ and is several times lower if one considers pumping via transitions in the stretching modes. The details of calculations are given in Appendix B. A possible radiative pumping via rotational transitions due to the background radiation field, including an IR source, is also found to be unimportant for our sources (Appendix B).

Chemistry. As both the opacity and molecular line excitation effects are found to be insufficient, the observed variations in molecular intensity to continuum flux ratios could mainly be connected with abundance variations of these molecules. Moreover, for different clumps within G285.26 and G291.27, the $\mathrm{N}_{2} \mathrm{H}^{+}$abundances are found to vary by an order of magnitude (see Sect. 4.2). High CS-to- $\mathrm{N}_{2} \mathrm{H}^{+}$intensity ratios have previously been found towards some starless clumps, for example, in S68N (Williams \& Myers 1999) and in Perseus (Olmi et al. 2005). They can be explained by $\mathrm{N}_{2} \mathrm{H}^{+}$underabundance that occurs at early phases of core evolution (Bergin et al. 1997). However, the high CS-to- $\mathrm{N}_{2} \mathrm{H}^{+}$intensity ratios in our cores are observed in the vicinity of IRAS sources, indicating that they are evolved regions. The drop in the $\mathrm{N}_{2} \mathrm{H}^{+}$intensity towards YSOs has been observed previously in Orion (Ungerechts et al. 1997) and Cepheus A (Bottinelli \& Williams 2004). Hot-core chemical models (e.g. Nomura \& Millar 2004) where ultraviolet radiation from embedded stars leads to the evaporation of grain mantles and CS enhancement, cannot explain why $\mathrm{N}_{2} \mathrm{H}^{+}$intensities do not correlate with gas column density in these regions.

Recently Lintott et al. (2005) have proposed an alternative model where CS to $\mathrm{N}_{2} \mathrm{H}^{+}$abundance ratio becomes enhanced due to higher collapse rates compared to a free-fall regime (accelerated collapse). As a result, high densities are achieved before $\mathrm{CS}$, and the molecules responsible for $\mathrm{N}_{2} \mathrm{H}^{+}$removal are freezing onto grains. Due to gradients of excitation temperatures, however, systematic collapse motions predicted by this model should give asymmetric or self-absorbed profiles of optically thick lines (e.g. CS(2-1)), which are not observed in most of the sources. Normalized velocity differences (Sect. 5.4) have not indicated such motions towards CS peaks in our sources according to the Mardones et al. (1997) criterion. If the sources consist of a large number of small, unresolved clumps with a low volumefilling factor, as implied in Sect. 4.2, the observed CS lines could have low effective optical depth and nearly symmetric profiles. In this case systematic collapse motions could be detected as additional line broadening towards core centers. The enhanced CS(5-4) line widths were detected towards intensity peaks in several sources (Sect. 5.4) where the $I\left(\mathrm{~N}_{2} \mathrm{H}^{+}\right) / F_{1.2}$ ratios are low. If the CS lines are actually not optically thick, these enhancements could imply some dynamical processes (e.g. collapse motions) in gas traced by CS. In order to decide whether or not the accelerated collapse model could be applied to these cores, one needs to conduct detailed radiative transfer calculations in comparison with observational data including other molecular tracers (e.g. $\mathrm{HCO}^{+}, \mathrm{NH}_{3}, \mathrm{SO}$ ).

Another possible explanation of the $\mathrm{N}_{2} \mathrm{H}^{+}$abundance drop can be related to its dissociative recombination. Earlier it was assumed that dissociative recombination of $\mathrm{N}_{2} \mathrm{H}^{+}$leads primarily to the formation of molecular nitrogen, which can form $\mathrm{N}_{2} \mathrm{H}^{+}$ again via reaction with $\mathrm{H}_{3}^{+}$. However, Geppert et al. (2004) find that another channel that leads to formation of the $\mathrm{NH}$ and $\mathrm{H}$ molecules dominates. Perhaps this could be why the $\mathrm{N}_{2} \mathrm{H}^{+}$abundance drops in vicinities of luminous YSOs. It is well known that UV radiation penetrates deep into dense molecular clouds, probably due to their clumpy structure (e.g. Meixner et al. 1992; Meixner \& Tielens 1993). Therefore, one can expect an enhanced ionization in the neighborhood of luminous young stars with a high UV flux, on scales comparable to those discussed here. However, published results of ionization fraction in massive cores do not support this view (Bergin et al. 1999). On the other hand, preliminary estimates of this fraction from our measurements for similar sources in the northern sky do show noticeable variations within the sources (Zinchenko et al, in preparation). A conclusive decision on the role of this factor in the observed chemical differentiation needs further investigation, including chemical modeling.

\section{Conclusions}

In order to get reliable information on the density and chemical structure of the dense cores associated with high-mass starforming regions, twelve objects from the southern hemisphere were mapped in the $\mathrm{CS}(5-4)$ line and in dust continuum at $1.2 \mathrm{~mm}$. We compared CS(5-4) and continuum data with each other, as well as with the $\mathrm{N}_{2} \mathrm{H}^{+}(1-0)$ (Paper I) and the CS(2-1) (Zinchenko et al. 1995) data. Besides, the physical parameters 
of the cores were derived from the $\mathrm{CS}(5-4)$ and the continuum data.

The results can be summarized as follows:

1. Most of the maps have several emission peaks (clumps). The mean sizes of 17 clumps with counterparts in the continuum and CS are 0.30(0.06) pc (continuum) and 0.51(0.07) pc (CS). The CS virial masses lie in the range $\sim 140-1630 M_{\odot}$. For the clumps with IRAS sources, we derived dust temperatures of 24-35 K, masses of 90-6900 $M_{\odot}$, molecular hydrogen column densities of $(0.7-12.0) \times 10^{23} \mathrm{~cm}^{-2}$, and luminosities of $(0.6-46.0) \times 10^{4} L_{\odot}$.

2. Using the CS(5-4) and the CS(2-1) data (Zinchenko et al. 1995), LVG densities in eight sources were calculated. Densities towards the CS peaks within the $50^{\prime \prime}$ beam $(0.56 \mathrm{pc}$ at $2.3 \mathrm{kpc}$, the average distance of our sample source) vary from source to source in the range $(3-40) \times 10^{5} \mathrm{~cm}^{-3}$. In four sources, the density falls by about an order of magnitude at $\sim 0.8-2 \mathrm{pc}$ from the CS peaks, which could be connected either to the existence of lower density clumps or density gradients within clumps. The masses calculated from LVG densities are higher than virial masses and than masses derived from continuum data, implying small-scale clumpiness of the cores. The CS abundances were calculated towards IRAS positions within the 50" Gaussian beam for eight sources: $X(\mathrm{CS})=(0.3-2.7) \times 10^{-9}$. The $\mathrm{N}_{2} \mathrm{H}^{+}$abundances for the same positions lie in the range $X\left(\mathrm{~N}_{2} \mathrm{H}^{+}\right)=$ $(0.3-4.4) \times 10^{-10}$.

3. For most of the objects, the CS and continuum peaks are close to the IRAS point source positions. The CS(5-4) integrated intensities correlate with continuum fluxes per beam almost linearly in all cases, while significant correlations between the CS(5-4) and the $\mathrm{N}_{2} \mathrm{H}^{+}(1-0)$ integrated intensities are only found in five sources. The highest CS to $\mathrm{N}_{2} \mathrm{H}^{+}$integrated intensity ratios $(\sim 10)$ are found in the vicinity of the most luminous IRAS sources. The lowest ratios $(\$ 0.1)$ are observed towards positions close to the centers of the $\mathrm{N}_{2} \mathrm{H}^{+}$clumps without CS counterparts and IRAS sources.

4. The study of spatial variations in the molecular integrated intensity ratios to continuum fluxes reveals that for most of the sources the $I\left(\mathrm{~N}_{2} \mathrm{H}^{+}\right) / F_{1.2}$ ratios drop towards $\mathrm{CS}$ and dust emission peaks near IRAS sources, most likely implying an underabundance of $\mathrm{N}_{2} \mathrm{H}^{+}$towards these peaks. The $I(\mathrm{CS}) / I\left(\mathrm{~N}_{2} \mathrm{H}^{+}\right)$ratios are clearly enhanced towards the three most luminous sources, whereas the $I\left(\mathrm{~N}_{2} \mathrm{H}^{+}\right) / F_{1.2}$ ratios drop towards these sources. Possible explanations of these results could involve dissociative recombination of $\mathrm{N}_{2} \mathrm{H}^{+}$or accelerated collapse processes. For $\mathrm{CS}(5-4)$ the $I(\mathrm{CS}) / F_{1.2}$ ratios show no clear trends with distance from the CS peaks, while for $\mathrm{CS}(2-1)$ such ratios drop towards these peaks, implying line saturation effects in several sources.

5. The analysis of normalized velocity differences between $\mathrm{CS}$ and $\mathrm{N}_{2} \mathrm{H}^{+}$lines has not revealed any indications of systematic motions in the sources towards CS peaks. A possible indication of infall motions has been found towards one position in G285.26. The CS(5-4) line widths in several sources have a tendency to decrease with distance from CS peaks, which could be connected both to optical depth effects and to enhanced dynamical activity towards the centers of the cores.

Acknowledgements. We are grateful to Alexander Lapinov for helpful discussions and suggestions and to Malcolm Walmsley for important comments. We would like to thank Marie-Lise Dubernet for sending us tables with the $\mathrm{N}_{2} \mathrm{H}^{+}-\mathrm{He}$ collisional rates and Luca Dore for providing reprints of the papers on the parameters of $\mathrm{N}_{2} \mathrm{H}^{+}$vibrational transitions. We would also like to thank the referee, Jorma Harju, for thoroughly examining the manuscript and for useful comments and recommendations that significantly improved the paper. We are also grateful to the language editor, J. Adams, for improving the grammer of the paper. The research made use of the SIMBAD database, operated by the CDS, Strasbourg, France. The work was supported by INTAS grant 99-1667 and the Russian Foundation for Basic Research grants 03-02-16307 and 06-02-16317 and by the Program "Extended objects in the Universe" of the Russian Academy of Sciences.

\section{Appendix A: The CS and $\mathrm{N}_{2} \mathrm{H}^{+}$LVG modeling results}

Using the LVG model, we examined the CS and $\mathrm{N}_{2} \mathrm{H}^{+}$excitation for a wide density range at distinct kinetic temperatures. When calculating the excitation of the $\mathrm{N}_{2} \mathrm{H}^{+}$unsplit rotation lines, we used the $\mathrm{N}_{2} \mathrm{H}^{+}-\mathrm{He}$ collisional rates from Daniel et al. (2005) (http://www . obspm.fr/basecol). In Fig. A.1 the $\mathrm{CS}(2-1), \mathrm{CS}(5-4)$, and $\mathrm{N}_{2} \mathrm{H}^{+}(1-0)$ brightness temperatures divided by $N / V$ (the ratio of gas column density to velocity at the boundary) are shown versus density at two values of kinetic temperature: $20 \mathrm{~K}$ and $40 \mathrm{~K}$. For convenience of presentation they are multiplied by the factor of $10^{14}$ and denoted as $P$. They can be compared with observed ratios of molecular line intensities to continuum fluxes (the latter are proportional to gas column density). The dependences of line optical depths $(\tau)$ on density are also shown. We set $d=0.5 \mathrm{pc}$ and $\Delta V=2.5 \mathrm{~km} \mathrm{~s}^{-1}$ as typical clump size and line width (Paper I) and $X\left(\mathrm{~N}_{2} \mathrm{H}^{+}\right)=5 \times 10^{-11}$ and $X(\mathrm{CS})=10^{-9}$ (which are close to the values for G285.26 from Table 6). These parameters enter the model as $N / V=n\left(\mathrm{H}_{2}\right)(2 d X) / \Delta V$.

The qualitative behavior of $P$ versus $n\left(\mathrm{H}_{2}\right)$ is similar for all the lines, yet, it reaches the maximum at higher densities for $\mathrm{CS}(5-4)$ than for $\mathrm{CS}(2-1)$ and $\mathrm{N}_{2} \mathrm{H}^{+}(1-0)$. The position of this maximum depends on $X(\mathrm{CS})$ : the lower $X(\mathrm{CS})$ the higher the density at which $P$ reaches maximum. After the maximum, $P$ decreases with density, approaching linear dependences (in logarithmic scale) due to the line saturation effect. As the CS(5-4) lines in our observations are not saturated, we only consider densities $\$ 10^{6} \mathrm{~cm}^{-3}$. Within the density range $10^{5}-10^{6} \mathrm{~cm}^{-3} P$ falls by a factor of $\lesssim 2$ for $\mathrm{N}_{2} \mathrm{H}^{+}(1-0)$ and by a factor $\sim 2.5-4$ for CS(2-1) (Fig. A.1, lower and upper panels). For the CS(2-1) lines, such a drop could be connected with saturation.

We have no data on the spatial distributions of kinetic temperatures in our sources, although one-point $\mathrm{CO}(1-0)$ (Zinchenko et al. 1995) and IRAS data probably do not imply temperatures significantly higher than $40 \mathrm{~K}$ towards CS and dust peaks for the regions with angular sizes $\sim 1^{\prime}$. If kinetic temperature rises from $20 \mathrm{~K}$ to $40 \mathrm{~K}$ towards the density peaks, $P$ decreases more slowly with the resulting decreasing factor of 1.3. Note that dust temperature gradients from $20 \mathrm{~K}$ to $40 \mathrm{~K}$ can reduce the observed $I\left(\mathrm{~N}_{2} \mathrm{H}^{+}\right) / F$ ratios by 2.3 times. Thus, one could expect that within the density range $10^{5}-10^{6} \mathrm{~cm}^{-3}$ the $I\left(\mathrm{~N}_{2} \mathrm{H}^{+}\right) / F$ ratios could drop by a factor not more than 3 as $T_{\mathrm{KIN}}$ and $T_{\mathrm{d}}$ rise from $20 \mathrm{~K}$ to $40 \mathrm{~K}$.

\section{Appendix B: Infrared pumping and excitation of $\mathrm{N}_{2} \mathrm{H}^{+}$}

Carroll \& Goldsmith (1981) derived the following criterion for infrared pumping to have an influence on molecular rotation spectra:

$f \exp \left(-\frac{h v}{k T_{s}}\right) \geq \frac{A_{\text {rot }}}{A_{\text {vibr }}}$ 


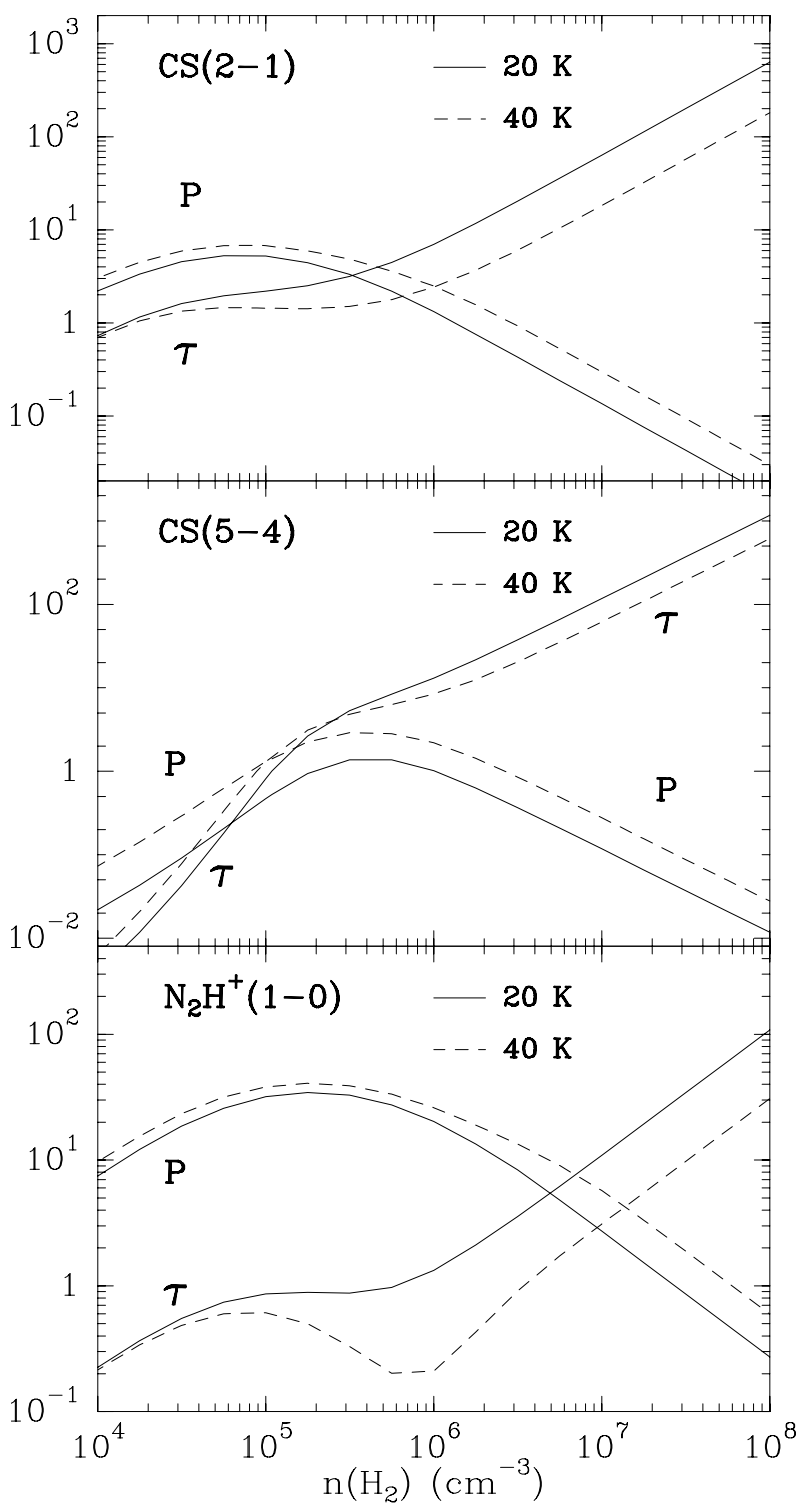

Fig. A.1. The results of LVG modeling: $\mathrm{CS}(2-1), \mathrm{CS}(5-4)$ and $\mathrm{N}_{2} \mathrm{H}^{+}(1-$ $0)$ brightness temperatures multiplied by the factor $10^{14}(N / V)^{-1}(P)$ versus density at two values of kinetic temperature. The dependences of line optical depths $(\tau)$ versus density are also shown.

where $f$ is a dilution factor of the pumping source that radiates at the temperature $T_{s} ; A_{\text {rot }}$ and $A_{\text {vibr }}$ are the Einstein spontaneous rates for rotational and vibrational transitions, respectively; $v$ is the frequency of the vibrational transition; $h$ and $k$ are the Plank and Boltzmann constants, respectively. Taking $f \approx R^{2} / 4 r^{2}$, where $R$ is the radius of the dust source, $r$ is the distance from the source, and $T_{s}(\mathrm{~K}) \approx 51\left(2 \times 10^{17} \mathrm{~cm}\right)^{1 / 3} R^{-1 / 3}($ Scoville \& Kwan 1976), one obtains:

$r \leq \frac{R}{2} \sqrt{\frac{A_{\text {vibr }}}{A_{\text {rot }}}} \exp \left(-\frac{h v}{k} \frac{1}{102} \frac{R^{1 / 3}}{\left(2 \times 10^{17}\right)^{1 / 3}}\right)$.

The right side of this expression peaks at some $R_{\mathrm{MAX}}$, corresponding to:

$r_{\text {MAX }}(\mathrm{cm}) \approx 2.87 \times 10^{24} \exp (-3)\left(\frac{h v}{k}\right)^{-3} \sqrt{\frac{A_{\text {vibr }}}{A_{\text {rot }}}}$,

so that the pumping could be effective at distances not higher than this value. For the $\mathrm{N}_{2} \mathrm{H}^{+}$molecule with $\mu=3.4 \mathrm{D}$ and $v_{\text {rot }}(1-0)=93.174 \mathrm{GHz}$ (Pickett et al. 1998), $A_{\text {rot }} \approx$ $3.6 \times 10^{-5} \mathrm{~s}^{-1}$. For the first vibrational stretching mode with $v_{1}=3234 \mathrm{~cm}^{-1}$ and $A_{\text {vibr }}=857 \mathrm{~s}^{-1}$ (Botshwina 1984), $r_{\text {MAX }} \approx 6.9 \times 10^{15} \mathrm{~cm} \approx 2 \times 10^{-3}$ pc. For the second bending mode with $v_{2}=698.6 \mathrm{~cm}^{-1}$ (Owrutsky et al. 1986), the lifetime of the excited state is $\tau=123.1 \mathrm{~ms}$ (Heninger et al. 2003) corresponding to $A_{\text {vibr }}=8.1 \mathrm{~s}^{-1}$ and leading to $r_{\mathrm{MAX}} \approx$ $6.6 \times 10^{16} \mathrm{~cm} \approx 2 \times 10^{-2} \mathrm{pc}$. For the third stretching mode with $v_{3}=2254 \mathrm{~cm}^{-1}$ (Botshwina 1984), $\tau=530.1 \mathrm{~ms}$ (Heninger et al. 2003) corresponding to $A_{\text {vibr }}=1.9 \mathrm{~s}^{-1}$ and leading to $r_{\mathrm{MAX}} \approx 9.5 \times 10^{14} \mathrm{~cm} \approx 3 \times 10^{-4} \mathrm{pc}$.

A possible radiative pumping via rotational transitions due to the background radiation field, including an IR source, is effective only when the source optical depth at these frequencies is higher than unity. From our continuum data, it is easy to estimate the peak optical depth at $1.2 \mathrm{~mm}$ averaged over the telescope beam in the optically thin case, $\tau=F \Omega^{-1} B_{v}(T)^{-1}$, where $F$ is the peak flux per beam, $\Omega$ the beam solid angle, $B_{v}(T)$ the Plank function at frequency $v$ and temperature $T$. Thus, for the strongest continuum source of our sample, G291.27, with peak flux $F=18.8 \mathrm{Jy} \mathrm{beam}^{-1}$ and $T=25 \mathrm{~K}, \tau \approx 0.05$ averaged over a region with a linear size $d \sim 0.3 \mathrm{pc}$. Taking a power-law index of the column density-radius dependence of -1 , which corresponds to the density-radius power-law index of -2 , one finds that in G291.27 the size of the region, where mean optical depth at $1.2 \mathrm{~mm}$ exceeds unity, is $d \sim 0.015 \mathrm{pc}$. Without averaging, the size of the optically thick region should be lower. Our calculations of $\mathrm{CS}$ and $\mathrm{N}_{2} \mathrm{H}^{+}$excitation that assume that infrared source radiates as a blackbody with temperature $T_{\mathrm{IR}}=100 \mathrm{~K}$ (close to the hot dust temperature component of the sample sources on average, see Sect. 4.1) show that the dilution factor for the pumping source should be $f \gtrsim 10^{-3}$ in order to produce a noticeable effect on the level populations. Therefore, the radius of affected region in $\mathrm{G} 291.27$ at $1.2 \mathrm{~mm}$ is $r=R /(2 \sqrt{f})=d /(4 \sqrt{f}) \lesssim 0.1 \mathrm{pc}$. The frequency of the $\mathrm{N}_{2} \mathrm{H}^{+}(2-1)$ transition that mainly affects the $J=1$ level population is about 1.5 times lower, therefore, the optical depth and the radius of the affected region should be 1.5-2 times smaller (depending on the power-law index of the dust optical depth-frequency dependence). For the other sources, this radius should be even smaller due to lower optical depths and lower distances in some cases.

\section{References}

Aikawa, Y., Ohashi, N., \& Herbst, E. 2003, ApJ, 593, 906 Bergin, E. A., Goldsmith, P. F., Snell, R. L., \& Langer, W. D. 1997, ApJ, 482, 285

Bergin, E. A., \& Langer, W. D. 1997, ApJ, 486, 316

Bergin, E. A., Plume, R., Williams, J. P., \& Myers, P. C. 1999, ApJ, 512, 724

Botschwina, P. 1984, Chem. Phys. Lett., 107, 535

Bottinelli, S., \& Williams, J. P. 2004, A\&A, 421, 1113

Braine, J., Krügel, E., Sievers, A., \& Wielebinski, R. 1995, A\&A, 295, L55

Brand, J., \& Blitz, L. 1993, A\&A, 275, 67

Carroll, T. J., \& Goldsmith, P. F. 1981, ApJ, 245, 891

Daniel, F., Dubernet, M.-L., Meuwly, M., Cernicharo, J., \& Pagani, L. 2005, MNRAS, 363, 1083

Doty, S. S., \& Leung, C. M. 1994, ApJ, 424, 729

Faundez, S., Bronfman, L., Garay, G., et al. 2004, A\&A, 426, 97

Geppert, W. D., Thomas, R., Semaniak, J., et al. 2004, ApJ, 609, 459

Heninger, M., Lauvergnat, D., Lemaire, J., et al. 2003, Int. J. Mass. Spectrom., 223-224, 669

Jijina, J., Myers, P. C., \& Adams, F. C. 1999, ApJS, 125, 161

Juvela, M. 1996, A\&AS, 118, 191

Juvela, M. 1998, A\&A, 329, 659

Lee, C. W., Myers, P. C., \& Tafalla, M. 1999, ApJ, 526, 788

Lee, C. W., Myers, P. C., \& Tafalla, M. 2001, ApJS, 136, 703

Li, Z.-Y., Shematovich, V. I., Wiebe, D. S., \& Shustov, B. M. 2002, ApJ, 569, 792 
Lintott, C. J., Viti, S., Rawlings, J. M. C., et al. 2005, ApJ, 620, 795 Mardones, D., Myers, P. C., Tafalla, M., et al. 1997, ApJ, 489, 719 McCutcheon, W. H., Sandell, G., Matthews, H. E., et al. 2000, MNRAS, 316, 152

Meixner, M., \& Tielens, A. G. G. M. 1993, ApJ, 405, 216

Meixner, M., Haas, M. R., Tielens, A. G. G. M., Erickson, E. F., \& Werner, M. 1992, ApJ, 390, 499

Motte, F., André, P., \& Neri, R. 1998, A\&A, 336, 150

Mozurkewich, D., Schwartz, P. R., \& Smith, H. A. 1986, ApJ, 311, 371

Mueller, K. E., Shirley, Y. L., Evans II, N. J., \& Jacobson, H. R. 2002, ApJS, 143,469

Neckel, T. 1978, A\&A, 69, 51

Nomura, H., \& Millar, T. J. 2004, A\&A, 414, 409

Olmi, L., Testi, L., \& Sargent, A. I. 2005, A\&A, 431, 253

Ossenkopf, V., \& Henning, T. 1994, A\&A, 291, 943

Owrutsky, J. C., Gudeman, C. S., Martner, C. C., et al. 1986, J. Chem. Phys., 84, 605

Pickett, H. M., Poynter, R. L., Cohen, E. A., et al. 1998, J. Quant. Spect. Rad. Trans., 60, 883
Pirogov, L. E., \& Zinchenko, I. I. 1998, AZh, 75, 14 (Astron. Rep., 42, 11) Pirogov, L., Zinchenko, I., Caselli, P., Johansson, L. E. B., \& Myers, P. C. 2003, A\&A, 405, 639 (Paper I)

Plume, R., Jaffe, D. T., Evans II, N. J., Martin-Pintado, J., \& Gomez-Gonzalez, J. ApJ, 476, 730

Scoville, N. Z., \& Kwan, J. 1976, ApJ, 206, 718

Shematovich, V. I., Wiebe, D. S., Shustov, B. M., \& Li, Z.-Y. 2003, ApJ, 588, 894

Shirley, Y. L., Evans II, N. J., Young, K. E., Knez, C., \& Jaffe, D. T. 2003, ApJS, 149,375

Tafalla, M., Myers, P. C., Caselli, P., Walmsley, C. M., \& Comito, C. 2002, ApJ, 569,815

Turner, B. E., Chan, K., Green, S., \& Lubowich, D. A. 1992, ApJ, 399, 114

Ungerechts, H., Bergin, E. A., Goldsmith, P. F., et al. 1997, ApJ, 482, 245

van Dishoeck, E. F. 2004, ARA\&A, 42, 119

Williams, J. P., \& Myers, P. C. 1999, ApJ, 518, L37

Zinchenko, I., Forsström, V., Lapinov, A., \& Mattila, K. 1994, A\&A, 288, 601

Zinchenko, I., Mattila, K., \& Toriseva, M. 1995, A\&AS, 111, 95

Zinchenko, I., Pirogov, L., \& Toriseva, M. 1998, A\&AS, 133, 337 